\title{
Space-charge impedance of rf-shielding wires with external ceramic and conducting pipes
}

\author{
Tai-Sen F. Wang and Sergey S. Kurennoy \\ Los Alamos National Laboratory, Los Alamos, New Mexico 87545 \\ Robert L. Gluckstern \\ University of Maryland, College Park, Maryland 20742
}

(Received 23 July 2001; published 17 October 2001)

\begin{abstract}
We studied the electrostatic field due to a charged-particle beam with uniform particle density propagating inside an rf-shielding cage (rf cage) constructed from evenly spaced conducting wires. The beam and the rf cage are surrounded by a ceramic beam pipe positioned inside a conducting pipe concentric with the beam and the rf cage. The space-charge impedances in the long wavelength regime are investigated by considering the electrostatic fields due to the longitudinal and transverse perturbations on the density of the charged-particle beam. Shielding effects due to the rf cage are discussed and simple formulas are derived for estimating the space-charge impedances. Numerical examples are given for illustration. Comparisons between analytical estimates and the results produced by the field-solver computer program MAFIA show good agreement.
\end{abstract}

DOI: 10.1103/PhysRevSTAB.4.104201

PACS numbers: 29.27.Bd, 29.20.Lq, 41.20.-q

\section{INTRODUCTION}

An rf-shielding cage, or an rf cage, used in an accelerator or storage ring is a cagelike structure made of perfectly conducting wires or rods parallel to the direction of the circulating charged particle beam [1]. The conducting wires of the cage are arranged to surround the beam to create an electromagnetically shielded environment for the beam. This type or similar types of devices together with ceramic beam pipes have been implemented [1] and planned [2-4] or are being planned [5] in some high-intensity rapid-cycling proton synchrotrons. One of the main reasons for choosing the rf cage instead of a solid conducting beam pipe is to avoid excess eddy current that may be induced on the beam pipe by the fast-changing magnetic field. In high-current accelerators, the interaction between the charged particle beam and the surrounding environment, characterized by the "coupling impedance" [6,7], plays an important role in the stability of the circulating beam. To avoid any collective instability, it is important to reduce the coupling impedance when the beam intensity is increased. Another reason for selecting the rf cage emerges when the impedance consideration becomes critical. It is easier to vary the cross section of an rf cage in order to reduce the coupling impedance than to change the cross section of a solid pipe. In the long wavelength region, an appropriately designed rf cage can provide electromagnetic shielding similar to that of a solid beam pipe.

Although an rf cage was built and installed in an existing proton synchrotron over a decade ago [1], serious studies of the electromagnetic field of a charged particle beam propagating in an rf cage were not attempted until recently [8-13]. The need to understand the electromagnetic fields in an rf-cage environment is prompted by several highintensity rapid-cycling synchrotrons proposed recently, as mentioned above. The purpose of this work is to inves- tigate the space-charge impedance of an rf cage mounted inside a ceramic and a conducting beam pipe. The analysis of the full electromagnetic field due to a beam traveling inside an rf cage is complicated by the details of the geometry of the cage and boundary conditions. However, in the long wavelength regime, electrostatic fields can be used. The studies presented in this paper will exploit such an approximation. We will solve the Poisson equation to obtain the electrostatic fields due to a perturbed beam traversing an rf cage. Then, we will derive simple formulas for computing both the longitudinal and transverse coupling impedances in the long wavelength regime. These formulas can be used as a guide in designing rf cages. Numerical examples will be given to illustrate the effect of $\mathrm{rf}$ shielding and the effect due to the ceramic pipe. Comparisons between analytical estimates and the results produced by the field-solver computer program MAFIA [14] will be provided.

Since the mathematical calculation involved in deriving the impedances is complicated and very lengthy, we will include the derivation of the perturbed field in the appendices and summarize the results in Sec. III. As the algebra is greatly simplified without the ceramic pipe, hence the details of deriving the perturbed field for a simpler case of no ceramic pipe will be presented in Appendix A to illustrate the procedures. The derivation of the perturbed field including the effects of a ceramic beam pipe will be outlined in Appendix B. Readers who are interested only in applying the final results for the impedances can skip Sec. III.

\section{THEORETICAL MODEL}

The cross section of the system considered here is shown in Fig. 1. A beam having a circular cross section of radius $r_{b}$ and a uniform charge distribution is propagating inside 


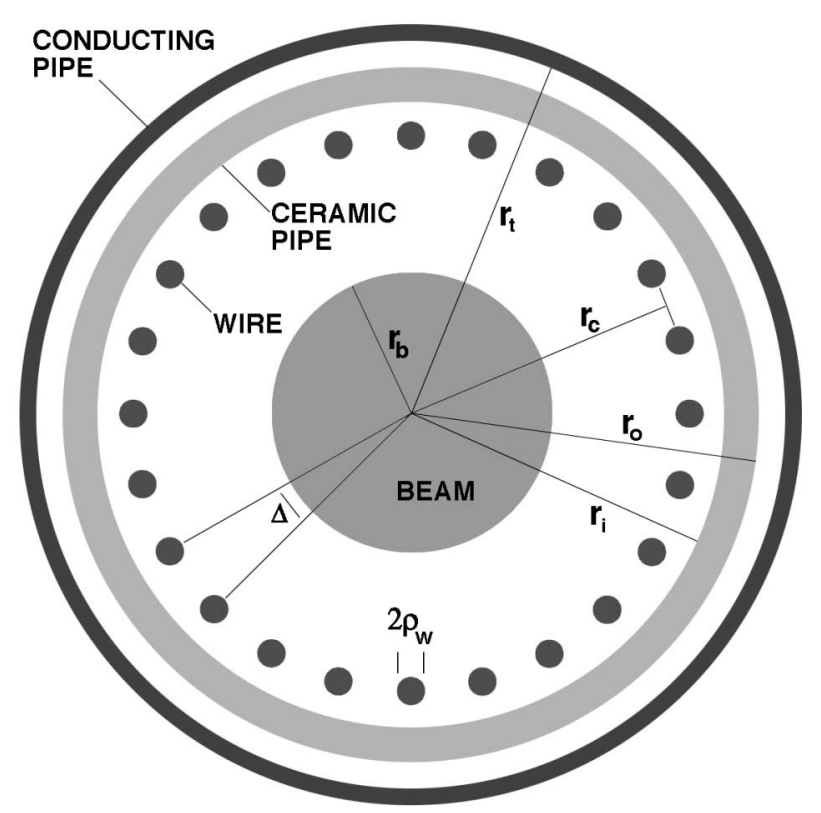

FIG. 1. Cross-sectional view of a beam inside an rf cage and the ceramic beam pipe and the external conducting beam pipe. $r_{t}, r_{c}$, and $r_{b}$ are the radii of the conducting beam pipe, the rf cage, and the beam, respectively. $r_{o}$ and $r_{i}$ are the external and the internal radii of the ceramic beam pipe, respectively. $\Delta$ is the angle subtended by two adjacent wires, and $\rho_{w}$ is the radius of a wire.

an rf cage composed of $N$ conducting wires parallel to the beam. The beam and the rf cage are surrounded by a ceramic beam pipe which is enclosed inside a conducting beam pipe with radius $r_{t}$. The external and the internal radii of the ceramic beam pipe are $r_{o}$ and $r_{i}$, respectively. We assume the ceramic has a uniform and isotropic permittivity $\epsilon$. For simplicity, we shall limit our discussion to the geometry in which wires are evenly distributed over a circle; the conducting and the ceramic pipes as well as the rf cage are positioned concentric with the beam. The radius of the rf cage, measured from the center of the cage to the centers of wires, is $r_{c}$. The pipe and wires are electrically grounded and all wires have the same circular cross section of radius $\rho_{w}$. The studies in the following will be focused on the regime of $\rho_{w} \ll r_{c}$ and $N \gg 1$.

A cylindrical coordinate system $(r, \theta, z)$ is chosen such that the $z$ axis coincides with the central axis of the beam. We call this coordinate system the "beam coordinate system" or the "global coordinate system." In order to make it convenient to describe the electric field near an individual wire, we also use another cylindrical coordinate system

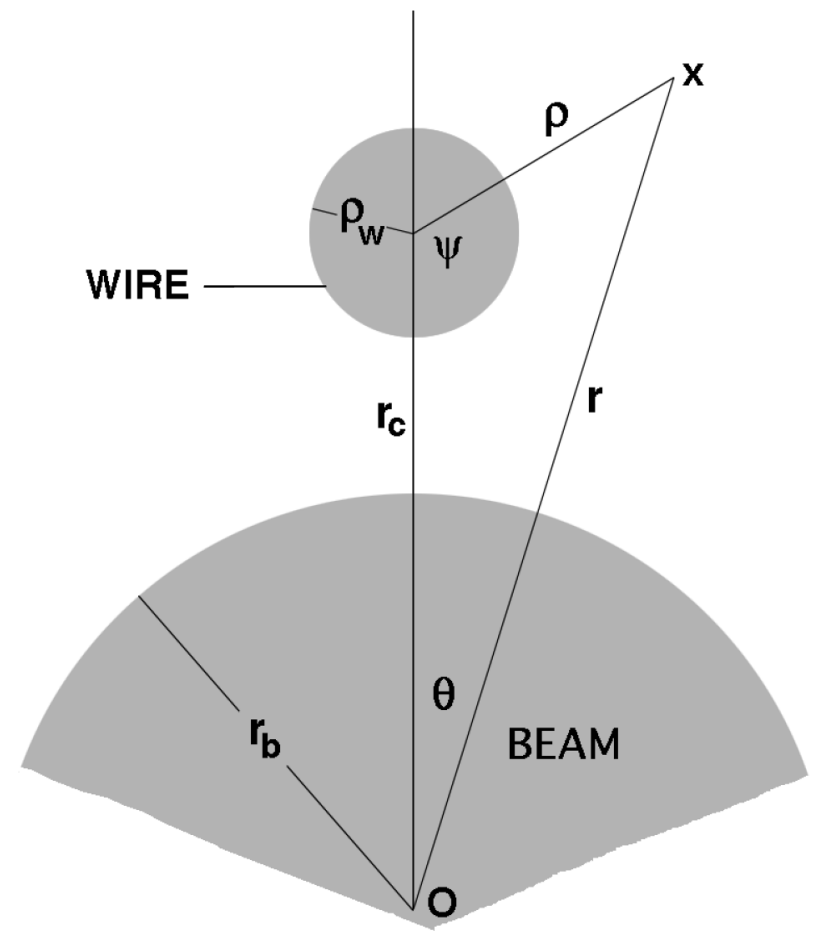

FIG. 2. The local and the global coordinates adopted in this study. The origins of the local and the global coordinates are located at the center of the beam and the center of a wire, respectively. The positive directions of $\psi$ and $\theta$ go counterclockwise and clockwise, respectively.

$(\rho, \psi, z)$ in which the $z$ axis coincides with the central axis of a wire, as shown in Fig. 2. This "local coordinate system" will also be referred to as the "wire coordinate system" in the following.

\section{PERTURBED FIELDS}

The analysis proceeds by first obtaining the electrostatic potential due to a perturbation in a beam propagating inside the rf cage described in the previous section. The longitudinal and transverse perturbations of the beam charge density will be considered separately in the following.

\section{A. Field due to a longitudinal perturbation}

Here, we will concentrate on the electrostatic potential due to a longitudinal charge-density perturbation in the beam that varies in the $z$ direction according to $e^{i k z}$, where $k$ is the wave number of the perturbation. The Poisson equation we want to solve is

$$
\frac{1}{r} \frac{\partial}{\partial r}\left(r \frac{\partial \Phi}{\partial r}\right)+\frac{1}{r^{2}} \frac{\partial^{2} \Phi}{\partial \theta^{2}}+\frac{\partial^{2} \Phi}{\partial z^{2}}= \begin{cases}0, & \text { for } r_{t}>r>r_{b} \\ -\left(\sigma / \epsilon_{o}\right) e^{i k z}, & \text { for } r \leq r_{b}\end{cases}
$$

where $\Phi$ is the electric potential, $\sigma$ is the volume charge density associated with the perturbation, and $\epsilon_{o}$ is the permittivity of free space. We are interested in the solution of Eq. (1) in the region where $k r_{t} \ll 1$. For mathematical convenience, 
the space inside the conducting pipe is divided into four zones: zone I in $r \leq r_{b}$, zone II in $r_{b} \leq r \leq r_{i}$, zone III in $r_{i} \leq r \leq r_{o}$, and zone IV in $r_{o} \leq r \leq r_{t}$. We assume $\Phi=0$ at $r=r_{t}$. The boundary conditions at $r_{i}$ are $\epsilon_{o} \partial \Phi_{\mathrm{II}} / \partial r=\epsilon \partial \Phi_{\mathrm{III}} / \partial r, \partial \Phi_{\mathrm{II}} / \partial z=\partial \Phi_{\mathrm{III}} / \partial z$, and $\partial \Phi_{\mathrm{II}} / \partial \theta=\partial \Phi_{\mathrm{III}} / \partial \theta$, where the subscripts of $\Phi$ indicate the zones considered. Similarly, the boundary conditions at $r_{o}$ are $\epsilon \partial \Phi_{\mathrm{III}} / \partial r=\epsilon_{o} \partial \Phi_{\mathrm{IV}} / \partial r$, $\partial \Phi_{\text {III }} / \partial z=\partial \Phi_{\text {IV }} / \partial z$, and $\partial \Phi_{\text {III }} / \partial \theta=\partial \Phi_{\text {IV }} / \partial \theta$.

The closed-form solution of Eq. (1) appears to be inaccessible. However, in the regime of $k \rho_{w} \ll k r_{c}<$ $k r_{t} \ll 1$, it is possible to find a solution expanded in powers of $h_{n}\left(k \rho_{w}\right)$, where

$$
h_{n}(x)=I_{n}(x) / K_{n}(x),
$$

and where $I_{n}(x)$ and $K_{n}(x)$ are the $n$th order modified Bessel functions of the first kind and the second kind, respectively. The solutions of $\Phi_{I I}, \Phi_{I I I}$, and $\Phi_{I V}$ are not directly required for calculating the space-charge impedance, except for field matching at zone boundaries in determining the solution of $\Phi_{\mathrm{I}}$ needed to obtain the impedance. We therefore concentrate on the explicit solutions in the region of $r \leq r_{c}$ in the following. As discussed in Appendix B, if the coupling among the multipole fields due to the induced charges on wires is neglected, the solution for $\Phi$ in the lowest order of $h_{n}\left(k \rho_{w}\right)$ is

$$
\Phi \approx b_{\|}\left[K_{0}(k r)-S_{0} I_{0}(k r)\right] e^{i k z}+N \sum_{p=-\infty}^{\infty} \sum_{n=-\infty}^{\infty} C_{\mathrm{II} n}\left[K_{n+p N}\left(k r_{c}\right)-(-1)^{n} S_{p N} I_{n+p N}\left(k r_{c}\right)\right] I_{p N}(k r) e^{i p N \theta} e^{i k z},
$$

for $r_{c} \geq r \geq r_{b}$, and

$$
\begin{aligned}
\Phi \approx & \left\{\frac{\sigma}{\epsilon_{o} k^{2}}-I_{0}(k r) b_{\|}\left[S_{0}+\frac{1}{h_{1}\left(k r_{b}\right)}\right]\right\} e^{i k z} \\
& +N \sum_{p=-\infty}^{\infty} \sum_{n=-\infty}^{\infty} C_{\mathrm{II} n}\left[K_{n+p N}\left(k r_{c}\right)-(-1)^{n} S_{p N} I_{n+p N}\left(k r_{c}\right)\right] I_{p N}(k r) e^{i p N \theta} e^{i k z},
\end{aligned}
$$

for $r \leq r_{b}$, where

$$
\begin{gathered}
b_{\|}=\left(\frac{\sigma r_{b}}{\epsilon_{o} k}\right) I_{1}\left(k r_{b}\right), \\
S_{l}=\frac{\epsilon \lambda_{l} I_{l}^{\prime}\left(k r_{i}\right)+\left[\epsilon-\epsilon_{o}-\epsilon_{o} \lambda_{l} h_{l}\left(k r_{i}\right)\right] K_{l}^{\prime}\left(k r_{i}\right)}{\epsilon h_{l}\left(k r_{i}\right) K_{l}^{\prime}\left(k r_{i}\right)-\left[\epsilon_{o}-\lambda_{l}\left(\epsilon-\epsilon_{o}\right) h_{l}\left(k r_{i}\right)\right] I_{l}^{\prime}\left(k r_{i}\right)}, \\
\lambda_{l}=\frac{1-\hat{Y} k r_{o} K_{l}\left(k r_{o}\right)\left[I_{l}^{\prime}\left(k r_{o}\right)-h_{l}\left(k r_{t}\right) K_{l}^{\prime}\left(k r_{o}\right)\right]}{\hat{Y} k r_{o} I_{l}\left(k r_{o}\right)\left[I_{l}^{\prime}\left(k r_{o}\right)-h_{l}\left(k r_{t}\right) K_{l}^{\prime}\left(k r_{o}\right)\right]-h_{l}\left(k r_{t}\right)}, \\
\hat{\Upsilon}=1-\left(\epsilon_{o} / \epsilon\right), \\
C_{\| n}=1+(-1)^{n} h_{n}\left(k \rho_{w}\right) \sum_{\mu=1}^{N-1} e^{-i n \mu \Delta} K_{0}\left(k d_{\mu}\right)-h_{n}\left(k \rho_{w}\right) \sum_{j=-\infty}^{\infty} S_{j}\left[I_{n+j}\left(k r_{c}\right)\right]^{2}\left[1+(-1)^{n} \sum_{\mu=1}^{N-1} e^{i j \mu \Delta}\right],
\end{gathered}
$$

the prime indicates the derivative with respect to the argument, $\Delta=2 \pi / N$ is the angular separation between two adjacent wires, $d_{\mu}$ is the distance between the centers of the 0th and the $\mu$ th wires, and wires are numbered by their angular locations relative to an arbitrarily chosen 0th wire in counterclockwise direction.

When $\epsilon \rightarrow \epsilon_{o}$, we have $\hat{Y} \rightarrow 0, S_{l} \rightarrow 1 / h_{l}\left(k r_{t}\right)$, and the electric potential approaches the limit of no ceramic pipe (found in Appendix A and in earlier work [10]). Further, for $\epsilon=\epsilon_{o}$ and $r_{t} \rightarrow \infty$, one finds $h_{l}\left(k r_{t}\right) \rightarrow \infty$ and the electric potential approaches that of no external conducting pipe (studied previously [9]).

In the regime of $k r_{b} \ll 1$, the axial component of the electric field $E_{z}$ varies little over the beam cross section. Hence, for the purpose of investigating the longitudinal impedance, we shall concentrate on $E_{z}$ at the center of the beam. Thus, taking the gradient of the electric potential in Eq. (4) and evaluating $E_{z}$ at $r=0$ yields

$$
\left.E_{z}\right|_{r=0} \approx-i k N \sum_{n=-\infty}^{\infty} C_{\mathrm{II} n}\left[K_{n}\left(k r_{c}\right)-(-1)^{n} S_{0} I_{n}\left(k r_{c}\right)\right] e^{i k z}-i\left\{\frac{\sigma}{\epsilon_{o} k}-k b_{\|}\left[S_{0}+\frac{1}{h_{1}\left(k r_{b}\right)}\right]\right\} e^{i k z} \text {. }
$$


For a small wire diameter, we can further take the approximation by retaining only the $n=0$ term in the summation, i.e., by considering the monopole contribution from the induced charges on each wire only, to obtain

$$
\left.E_{z}\right|_{r=0} \approx \frac{i k N b_{\|} h_{0}\left(k \rho_{w}\right)}{G_{\| 0}}\left[K_{0}\left(k r_{c}\right)-S_{0} I_{0}\left(k r_{c}\right)\right]^{2} e^{i k z}-i\left\{\frac{\sigma}{\epsilon_{o} k}-k b_{\|}\left[S_{0}+\frac{1}{h_{1}\left(k r_{b}\right)}\right]\right\} e^{i k z}
$$

Using the addition theorem of Bessel function [15], Eq. (10) becomes

$$
\begin{aligned}
G_{\| 0}=1+h_{0}\left(k \rho_{w}\right)\{ & (N-1) K_{0}\left(k r_{c}\right) I_{0}\left(k r_{c}\right)-2 \sum_{q=1}^{N-1} K_{q}\left(k r_{c}\right) I_{q}\left(k r_{c}\right) \\
& \left.+2 \sum_{p=1}^{\infty}\left[(N-1) K_{p N}\left(k r_{c}\right) I_{p N}\left(k r_{c}\right)-\sum_{q=1}^{N-1} K_{q+p N}\left(k r_{c}\right) I_{q+p N}\left(k r_{c}\right)\right]\right\} \\
& -N h_{0}\left(k \rho_{w}\right) \sum_{p=-\infty}^{\infty} S_{p N}\left[I_{p N}\left(k r_{c}\right)\right]^{2} .
\end{aligned}
$$

For $N \gg 1$ and $k r_{c} \ll 1$, one can apply the small argument expansions of Bessel functions in Eqs. (6) and (13) to show that

$$
\begin{gathered}
S_{0} \approx-\ln \left(k r_{t}\right)+\Upsilon \ln \left(\frac{r_{o}}{r_{i}}\right), \\
S_{1} \approx \frac{-2 H_{1}}{N\left(k r_{c}\right)^{2}}, \\
S_{p N}\left[I_{p N}\left(k r_{c}\right)\right]^{2} \approx \frac{H_{p N}}{2 N},
\end{gathered}
$$

for $p>0$,

$$
\sum_{p=-\infty}^{\infty} S_{p N+1}\left[I_{p N+1}\left(k r_{c}\right)\right]^{2} \approx-\frac{H_{1}}{2}-\frac{T}{N}
$$

and

$$
\begin{aligned}
G_{\| 0} & \approx 1-h_{0}\left(k \rho_{w}\right)\left\{(N-1) \ln \left(k r_{c}\right)+\ln N+N \sum_{p=-\infty}^{\infty} S_{p N}\left[I_{p N}\left(k r_{c}\right)\right]^{2}\right\} \\
& \approx \frac{1}{\ln \left(k \rho_{w}\right)}\left[-N \ln \left(\frac{r_{t} r_{i}}{r_{c} r_{o}}\right)+\ln \left(\pi f_{w}\right)+N\left(\frac{\epsilon_{o}}{\epsilon}\right) \ln \left(\frac{r_{i}}{r_{o}}\right)+\sum_{p=1}^{\infty} H_{p N}\right],
\end{aligned}
$$

where

$$
\begin{gathered}
Y=1-\left(\epsilon / \epsilon_{o}\right), \\
H_{l}=\frac{N}{l}\left(\frac{r_{c}}{r_{i}}\right)^{2 l}\left\{\frac{\left(\epsilon-\epsilon_{o}\right)-\lambda_{l}\left[I_{l}\left(k r_{i}\right) / K_{l}\left(k r_{i}\right)\right]\left(\epsilon+\epsilon_{o}\right)}{\left(\epsilon+\epsilon_{o}\right)-\lambda_{l}\left[I_{l}\left(k r_{i}\right) / K_{l}\left(k r_{i}\right)\right]\left(\epsilon-\epsilon_{o}\right)}\right\} \\
\approx \frac{-N}{l}\left(\frac{r_{c}}{r_{i}}\right)^{2 l}\left\{\frac{\left(\epsilon_{o}+\epsilon\right)^{2}\left(\frac{r_{i}}{r_{t}}\right)^{l}-\left(\epsilon_{o}-\epsilon\right)^{2}\left(\frac{r_{o}^{2}}{r_{i} r_{t}}\right)^{l}+\left(\epsilon_{o}^{2}-\epsilon^{2}\right)\left[\left(\frac{r_{i} r_{t}}{r_{o}^{2}}\right)^{l}-\left(\frac{r_{t}}{r_{i}}\right)^{l}\right]}{\left(\epsilon+\epsilon_{o}\right)^{2}\left(\frac{r_{t}}{r_{i}}\right)^{l}-\left(\epsilon_{o}-\epsilon\right)^{2}\left(\frac{r_{i} r_{t}}{r_{o}^{2}}\right)^{l}+\left(\epsilon_{o}^{2}-\epsilon^{2}\right)\left[\left(\frac{r_{o}^{2}}{r_{i} r_{t}}\right)^{l}-\left(\frac{r_{i}}{r_{t}}\right)^{l}\right]}\right\}, \\
T=\frac{-1}{2} \sum_{p=1}^{\infty}\left(H_{p N+1}+H_{p N-1}\right),
\end{gathered}
$$

and

$$
f_{w}=\frac{\theta_{w}}{\Delta} \approx \frac{N \rho_{w}}{\pi r_{c}}
$$

is the "wire filling factor" defined as the ratio between the angle subtended by a wire in the beam coordinate system $\theta_{w}$ and $\Delta$. Substituting Eqs. (14) and (18) into Eq. (12), we can derive

$$
\left.E_{z}\right|_{r=0} \approx \frac{-i \sigma k r_{b}^{2}}{2 \epsilon_{o}}\left\{\frac{1}{2}+\ln \left(\frac{r_{c}}{r_{b}}\right)-\frac{\left[\ln \left(\frac{r_{c}}{r_{t}}\right)-\Upsilon \ln \left(\frac{r_{i}}{r_{0}}\right)\right]\left[\ln \left(\pi f_{w}\right)+\sum_{p=1}^{\infty} H_{p N}\right]}{N\left[\ln \left(\frac{r_{c}}{r_{t}}\right)-\Upsilon \ln \left(\frac{r_{i}}{r_{o}}\right)\right]+\ln \left(\pi f_{w}\right)+\sum_{p=1}^{\infty} H_{p N}}\right\} e^{i k z} .
$$


Note that, when $1 \ll l$ and $\left(r_{i} / r_{t}\right)^{2 l} \ll\left|\epsilon_{o}-\epsilon\right| /\left(\epsilon_{o}+\epsilon\right)$, the following approximations are valid:

$$
\begin{gathered}
H_{l} \approx \frac{N}{l}\left(\frac{\epsilon_{o}-\epsilon}{\epsilon_{o}+\epsilon}\right)\left(\frac{r_{c}}{r_{i}}\right)^{2 l}, \\
\sum_{p=1}^{\infty} H_{p N} \approx-\left(\frac{\epsilon_{o}-\epsilon}{\epsilon_{o}+\epsilon}\right) \ln \left[1-\left(\frac{r_{c}}{r_{i}}\right)^{2 N}\right],
\end{gathered}
$$

and

$$
T \approx \frac{\epsilon_{o}-\epsilon}{2\left(\epsilon_{o}+\epsilon\right)}\left[\left(\frac{r_{c}}{r_{t}}\right)^{2}+\left(\frac{r_{t}}{r_{c}}\right)^{2}\right] \ln \left[1-\left(\frac{r_{c}}{r_{t}}\right)^{2 N}\right] .
$$

These approximations can be helpful in the numerical computation of $E_{z}$ given in Eq. (23).

\section{B. Field due to a transverse perturbation}

The model of the perturbation studied here is a shell with surface charge density varying according to $e^{i k z} \cos \theta$. (We assume the beam or the global coordinate system is oriented in such a way that the maximal perturbed charge density is at the angle of $\theta=0$.) The appropriate Poisson equation is

$$
\frac{1}{r} \frac{\partial}{\partial r}\left(r \frac{\partial \Phi}{\partial r}\right)+\frac{1}{r^{2}} \frac{\partial^{2} \Phi}{\partial \theta^{2}}+\frac{\partial^{2} \Phi}{\partial z^{2}}=-\frac{\sigma \bar{d}}{\epsilon_{o}} \delta\left(r-r_{b}\right) e^{i k z} \cos \theta
$$

where $\bar{d}$ is the maximal displacement of the beam.

Here again, we will concentrate on the explicit solutions in the region of $r \leq r_{c}$. In Appendix B, we derived the following solution of Eq. (27):

$$
\begin{aligned}
\Phi \approx & 2 b_{\perp}\left[K_{1}(k r)-S_{1} I_{1}(k r)\right] \cos \theta e^{i k z} \\
& +2 N \sum_{p=-\infty}^{\infty} \sum_{n=-\infty}^{\infty} I_{p N+1}(k r) P_{\mathrm{II} n}\left[K_{n+p N+1}\left(k r_{c}\right)-(-1)^{n} S_{p N+1} I_{n+p N+1}\left(k r_{c}\right)\right] e^{i p N \theta} \cos \theta e^{i k z},
\end{aligned}
$$

for $r_{b} \leq r \leq r_{c}$, and

$$
\begin{aligned}
\Phi \approx & 2 b_{\perp}\left[\frac{1}{h_{1}\left(k r_{b}\right)}-S_{1}\right] I_{1}(k r) \cos \theta e^{i k z} \\
& +2 N \sum_{p=-\infty}^{\infty} \sum_{n=-\infty}^{\infty} I_{p N+1}(k r) P_{\mathrm{II} n}\left[K_{n+p N+1}\left(k r_{c}\right)-(-1)^{n} S_{p N+1} I_{n+p N+1}\left(k r_{c}\right)\right] e^{i p N \theta} \cos \theta e^{i k z},
\end{aligned}
$$

for $r \leq r_{b}$, where

$$
b_{\perp}=\left(\frac{\sigma \bar{d} r_{b}}{2 \epsilon_{o}}\right) I_{1}\left(k r_{b}\right),
$$

$h_{l}(x)$ and $S_{l}$ were defined in Eqs. (2) and (6), respectively,

$$
\begin{gathered}
P_{\mathrm{II} n}=-b_{\perp} h_{n}\left(k \rho_{w}\right)\left[K_{n+1}\left(k r_{c}\right)-S_{1} I_{n+1}\left(k r_{c}\right)\right] G_{\perp n}^{-1}, \\
G_{\perp n}=1+(-1)^{n} h_{n}\left(k \rho_{w}\right)\left\{\sum_{\mu=1}^{N-1} e^{-i(n-1) \mu \Delta} K_{0}\left(k d_{\mu}\right)-\sum_{j=-\infty}^{\infty} S_{j}\left[I_{j+n}\left(k r_{c}\right)\right]^{2}\left[1+(-1)^{n} \sum_{\mu=1}^{N-1} e^{i(j+1) \mu \Delta}\right]\right\},
\end{gathered}
$$

and $d_{j, m}$ is the distance between the centers of the $m$ th and $j$ th wires.

Taking the limit of $\epsilon \rightarrow \epsilon_{o}$ in Eqs. (28) and (29) yields the electric potential for the case of no ceramic pipe (obtained in Appendix A as well as in Ref. [10]). If the limit of $r_{t} \rightarrow \infty$ is also considered, we find the electric potential in the case of no ceramic and conducting pipes [9].

For studying the transverse impedance, we will focus on the transverse electric field $E_{y}$ at the beam center. By taking the gradient of the electric potential given in Eq. (29) and retaining the monopole $(n=0)$ term only, we have

$$
\left.E_{y}\right|_{r=0} \approx-b_{\perp} k\left[\frac{1}{h_{1}\left(k r_{b}\right)}-S_{1}\right] e^{i k z}-N k P_{\mathrm{II} 0}\left[K_{1}\left(k r_{c}\right)-S_{1} I_{1}\left(k r_{c}\right)\right] e^{i k z}
$$

where

$$
P_{\mathrm{II} 0} \approx-b_{\perp} h_{0}\left(k \rho_{w}\right)\left[K_{1}\left(k r_{c}\right)-S_{1} I_{1}\left(k r_{c}\right)\right] G_{\perp 0}^{-1},
$$


and

$$
G_{\perp 0} \approx 1+h_{0}\left(k \rho_{w}\right)\left\{N \sum_{l=-\infty}^{\infty} \sum_{p=-\infty}^{\infty} \delta_{|l|,|p N-1|} K_{l}\left(k r_{c}\right) I_{l}\left(k r_{c}\right)-\sum_{l=-\infty}^{\infty} K_{l}\left(k r_{c}\right) I_{l}\left(k r_{c}\right)-N \sum_{p=-\infty}^{\infty} S_{|p N+1|[}\left[I_{|p N+1|}\left(k r_{c}\right)\right]^{2}\right\} .
$$

Substituting Eqs. (15) and (17) into Eq. (35), and using the small argument expansions of Bessel functions, one can show that

$$
\begin{aligned}
G_{\perp 0} & \approx 1+h_{0}\left(k \rho_{w}\right)\left\{\frac{N}{2}+\ln \left(k r_{c}\right)-\ln N-N \sum_{p=-\infty}^{\infty} S_{|p N+1|}\left[I_{|p N+1|}\left(k r_{c}\right)\right]^{2}\right\} \\
& \approx 1+h_{0}\left(k \rho_{w}\right)\left[\frac{N}{2}+\ln \left(k r_{c}\right)-\ln N+\frac{H_{1}}{2 N}+\frac{T}{N}\right] .
\end{aligned}
$$

From this result and Eq. (34), we have

$$
P_{\mathrm{II} 0} \approx \frac{-2\left(b_{\perp} / k r_{c}\right)\left[1+\left(H_{1} / N\right)\right]}{N+H_{1}-2 \ln \left(\pi f_{w}\right)+2 T}
$$

where $H_{l}$ is given in Eq. (20). Substituting Eqs. (30), (15), and (37) into Eq. (33) yields

$$
\left.E_{y}\right|_{r=0} \approx-\left(\frac{\sigma \bar{d} r_{b}^{2}}{2 \epsilon_{o}}\right)\left\{\frac{1}{r_{b}^{2}}-\frac{1}{r_{c}^{2}}-\frac{2\left[\ln \left(\pi f_{w}\right)-T\right]\left(N+H_{1}\right)}{N r_{c}^{2}\left[N+H_{1}-2 \ln \left(\pi f_{w}\right)+2 T\right]}\right\} e^{i k z} .
$$

\section{SPACE-CHARGE IMPEDANCE}

We now study the space-charge impedance in the long wavelength regime, i.e., the regime of $k \rho_{w} \ll k r_{t} \ll 1$. The discussion here will concentrate on the field at the center of the beam $(r=0)$ given in Eqs. (23) and (38). It is important to recall that in arriving at these results we have retained only the most significant terms (the $n=0$ or the monopole terms) in the summations, and we have neglected the couplings among the multipole fields since they are negligibly small in the parameter range of our interest.

From the fields at the center of the beam given in Eqs. (23) and (38), we can infer that, for a circular accelerator or a storage ring, the longitudinal space-charge impedance $Z_{\|}$and the transverse space-charge impedance $Z_{\perp}$ are given by

$$
Z_{\|} \approx \frac{i n Z_{0}}{2 \beta \gamma^{2}}\left\{1+2 \ln \left(\frac{r_{c}}{r_{b}}\right)-\frac{2\left[\ln \left(\frac{r_{c}}{r_{t}}\right)-\left(1-\frac{\epsilon}{\epsilon_{o}}\right) \ln \left(\frac{r_{i}}{r_{o}}\right)\right]\left[\ln \left(\pi f_{w}\right)+\sum_{p=1}^{\infty} H_{p N}\right]}{N\left[\ln \left(\frac{r_{c}}{r_{t}}\right)-\left(1-\frac{\epsilon}{\epsilon_{o}}\right) \ln \left(\frac{r_{i}}{r_{o}}\right)\right]+\ln \left(\pi f_{w}\right)+\sum_{p=1}^{\infty} H_{p N}}\right\},
$$

and

$$
Z_{\perp} \approx\left(\frac{i R Z_{0}}{\beta^{2} \gamma^{2}}\right)\left\{\frac{1}{r_{b}^{2}}-\frac{1}{r_{c}^{2}}-\frac{2\left[\ln \left(\pi f_{w}\right)-T\right]\left(N+H_{1}\right)}{N r_{c}^{2}\left[N+H_{1}-2 \ln \left(\pi f_{w}\right)+2 T\right]}\right\}
$$

where $n$ is the harmonic number of the longitudinal perturbation around the ring, $Z_{0}=377 \Omega, \beta$ is the ratio of the speed of the beam particles to the speed of light, $\gamma=\left(1-\beta^{2}\right)^{-1 / 2}, R$ is the effective machine radius, $f_{w}$ was defined in Eq. (22); $H_{l}$ and $T$ are given in Eqs. (20) and (21), respectively. These impedances can be put into more convenient forms as

$$
Z_{\|} \approx \frac{i n Z_{o}}{2 \beta \gamma^{2}}\left\{\left[1+2 \ln \left(\frac{r_{c}}{r_{b}}\right)\right]\left(1-M_{\|}\right)+\left[1+2 \ln \left(\frac{r_{t}}{r_{b}}\right)\right] M_{\|}\right\}
$$

and

$$
Z_{\perp} \approx \frac{i R Z_{o}}{\beta^{2} \gamma^{2}}\left[\left(\frac{1}{r_{b}^{2}}-\frac{1}{r_{c}^{2}}\right)\left(1-M_{\perp}\right)+\left(\frac{1}{r_{b}^{2}}-\frac{1}{r_{t}^{2}}\right) M_{\perp}\right]
$$

where

$$
M_{\|}=\frac{\left[\ln \left(\frac{r_{c}}{r_{t}}\right)-\left(1-\frac{\epsilon}{\epsilon_{o}}\right) \ln \left(\frac{r_{i}}{r_{o}}\right)\right]\left[\ln \left(\pi f_{w}\right)+\sum_{p=1}^{\infty} H_{p N}\right]}{\ln \left(\frac{r_{c}}{r_{t}}\right)\left\{N\left[\ln \left(\frac{r_{c}}{r_{t}}\right)-\left(1-\frac{\epsilon}{\epsilon_{o}}\right) \ln \left(\frac{r_{i}}{r_{o}}\right)\right]+\ln \left(\pi f_{w}\right)+\sum_{p=1}^{\infty} H_{p N}\right\}},
$$

and

$$
M_{\perp}=\frac{-2\left(N+H_{1}\right)\left[\ln \left(\pi f_{w}\right)-T\right]}{N\left[1-\left(r_{c} / r_{t}\right)^{2}\right]\left[N+H_{1}-2 \ln \left(\pi f_{w}\right)+2 T\right]}
$$


Effective shielding by the rf cage is then achieved by requiring $M_{\|} \ll 1$ or $M_{\perp} \ll 1$, or, equivalently,

$$
N \ln \left(\frac{r_{t}}{r_{c}}\right) \gg-\ln \left(\pi f_{w}\right)+\ln \left[1-\left(\frac{r_{c}}{r t}\right)^{2 N}\right]
$$

for effective shielding of the longitudinal impedance, and

$$
N\left[1-\left(\frac{r_{c}}{r_{t}}\right)^{2}\right] \gg-2 \ln \left(\pi f_{w}\right)+2 T-\frac{4\left[\ln \left(\pi f_{w}\right)-T\right]^{2}}{N+H_{1}-2 \ln \left(\pi f_{w}\right)+2 T},
$$

for effective shielding of the transverse impedance. It is worthwhile to reiterate that, when $1 \ll l$ and $\left(r_{i} / r_{t}\right)^{2 l} \ll \mid \epsilon_{o}-$ $\epsilon \mid /\left(\epsilon_{o}+\epsilon\right)$, the approximations in Eqs. (24)-(26) can be used throughout Eqs. (39)-(46) for numerical estimates.

Equations (39)-(42) are valid in the limit of $N \gg 1$ and $k r_{t}=\omega r_{t} / v \ll 1$. In these limits, we expect the effect of the dielectric to be unimportant since the frequency will not be large enough to encounter resonant effects within the dielectric layer. We therefore obtain the results in the absence of the dielectric by setting $\epsilon=\epsilon_{o}$, or by setting $r_{i}=r_{o}$. In either case, we have

$$
M_{\|}=\frac{-\ln \left(\pi f_{w}\right)+\ln \left[1-\left(r_{c} / r_{t}\right)^{2 N}\right]}{N \ln \left(r_{t} / r_{c}\right)-\ln \left(\pi f_{w}\right)+\ln \left[1-\left(r_{c} / r_{t}\right)^{2 N}\right]},
$$

and

$$
M_{\perp}=\frac{-2 \ln \left(\pi f_{w}\right)+\left[\left(r_{c} / r_{t}\right)^{2}+\left(r_{t} / r_{c}\right)^{2}\right] \ln \left[1-\left(r_{c} / r_{t}\right)^{2 N}\right]}{N\left[1-\left(r_{c} / r_{t}\right)^{2}\right]-2 \ln \left(\pi f_{w}\right)+\left[\left(r_{c} / r_{t}\right)^{2}+\left(r_{t} / r_{c}\right)^{2}\right] \ln \left[1-\left(r_{c} / r_{t}\right)^{2 N}\right]}
$$

These results are consistent with those in earlier studies [10,13]. The condition for effective shielding of longitudinal impedance is the same as shown in Eq. (45), and the condition for effective shielding of transverse impedance is simplified to

$$
N\left[1-\left(\frac{r_{c}}{r_{t}}\right)^{2}\right] \gg-2 \ln \left(\pi f_{w}\right)+\left[\left(\frac{r_{c}}{r_{t}}\right)^{2}+\left(\frac{r_{t}}{r_{c}}\right)^{2}\right] \ln \left[1-\left(\frac{r_{c}}{r_{t}}\right)^{2 N}\right] .
$$

\section{NUMERICAL EXAMPLES}

As can be seen from Eqs. (41)-(44), there are several parameters involved in the impedances. We therefore focus on the numerical results for the quantities $M_{\|}$and $M_{\perp}$. Examples are presented in Figs. 3-6, where the values of $M_{\|}$and $M_{\perp}$ computed using Eqs. (43) and (44) are plotted as functions of the number of wires for various values of $r_{c} / r_{i}, r_{i} / r_{o}, r_{o} / r_{t}, f_{w}$, and $\epsilon / \epsilon_{o}$. As can be seen from these examples, the values of $M_{\|}$and $M_{\perp}$ decrease when the shielding is improved by increasing the number of wires, while the variation of the dielectric constant does not seem to make a significant effect. The only appreciable differences between the cases of $\epsilon=\epsilon_{o}$ and $\epsilon=10 \epsilon_{o}$ appear when $N<30$. This is partly because the ceramic pipe is placed outside the rf cage. We also observe that the values of $M_{\|}$and $M_{\perp}$ decrease with increasing wire size or $f_{w}$ when the shielding effect of the cage increases. Our results also show that the variation of the wire filling factor $f_{w}$ can make appreciable difference only when the number of wires is less than 75 . When the external conducting pipe is close to the ceramic pipe and $f_{w} \leq 0.1$, the dielectric pipe appears to have opposite effects on $M_{\|}$and $M_{\perp}$, as shown in Figs. 5 and 6.
In the practical parameter range, $N \gg 1$, $\left[1-\left(\epsilon / \epsilon_{o}\right)\right] \ln \left(r_{o} / r_{i}\right) \ll N$, and $r_{t}$ is always larger than

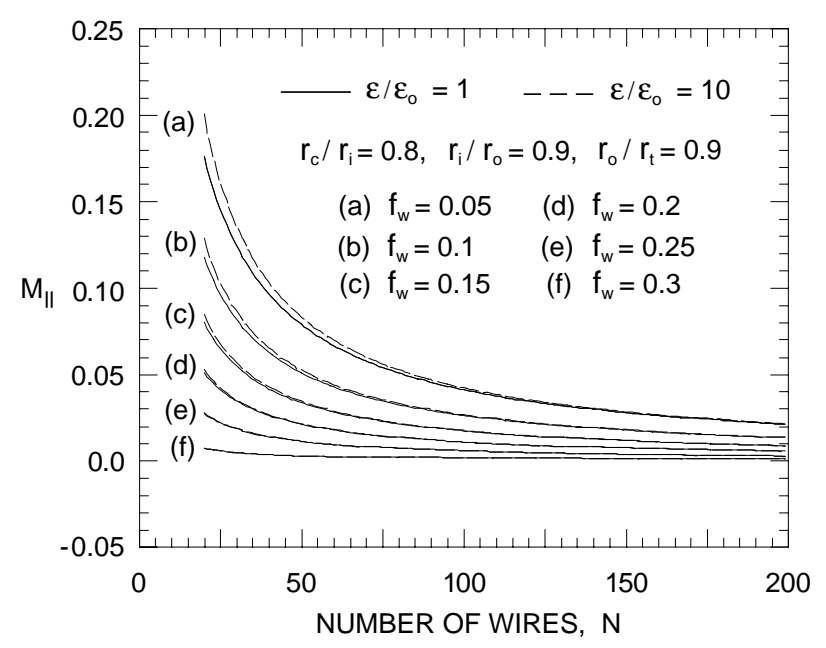

FIG. 3. $M_{\|}$as a function of the total number of wires $N$ for (a) $f_{w}=0.05$, (b) $f_{w}=0.1$, (c) $f_{w}=0.15$, (d) $f_{w}=0.2$, (e) $f_{w}=0.25$, and (f) $f_{w}=0.3$. Here, $M_{\|}$was calculated using Eq. (43) for $r_{c} / r_{i}=0.8, r_{i} / r_{o}=0.9$, and $r_{o} / r_{t}=0.9$. 


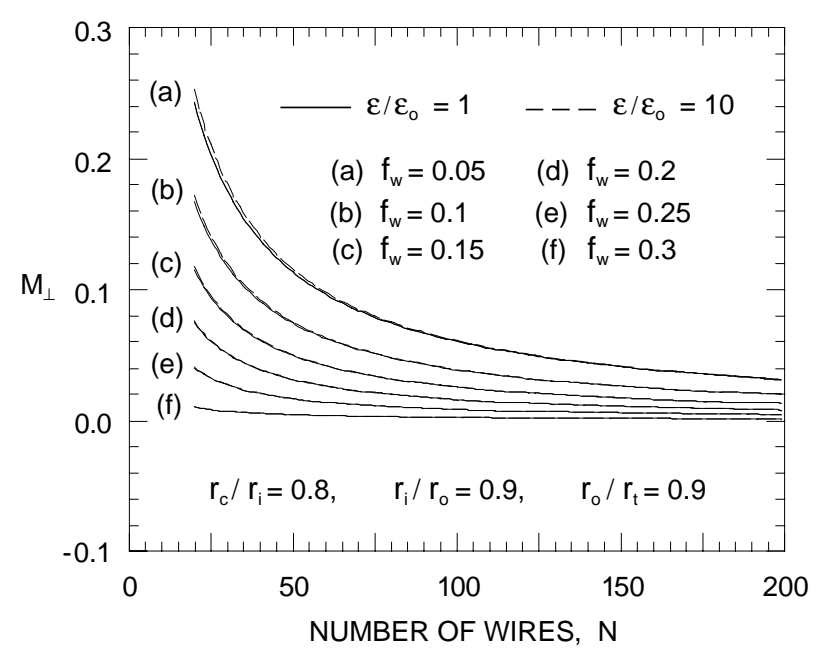

FIG. 4. $M_{\perp}$ as a function of the total number of wires $N$ for (a) $f_{w}=0.05$, (b) $f_{w}=0.1$, (c) $f_{w}=0.15$, (d) $f_{w}=0.2$, (e) $f_{w}=0.25$, and (f) $f_{w}=0.3$. Here, $M_{\perp}$ was calculated using Eq. (44) for $r_{c} / r_{i}=0.8, r_{i} / r_{o}=0.9$, and $r_{o} / r_{t}=0.9$.

$r_{c}$, it is easy to obtain the following approximations from Eqs. (43) and (44):

$$
R_{L}=\frac{N M_{\|}}{1-M_{\|}} \approx \frac{\ln \left(\pi f_{w}\right)}{\ln \left(r_{c} / r_{t}\right)}
$$

and

$$
R_{T}=\frac{N M_{\perp}}{1-M_{\perp}} \approx \frac{-2 \ln \left(\pi f_{w}\right)}{1-\left(r_{c} / r_{t}\right)^{2}} .
$$

Thus the quantities $R_{L}$ and $R_{T}$ mainly depend on $f_{w}$ and $r_{c} / r_{t}$ but not much on other parameters. It is therefore

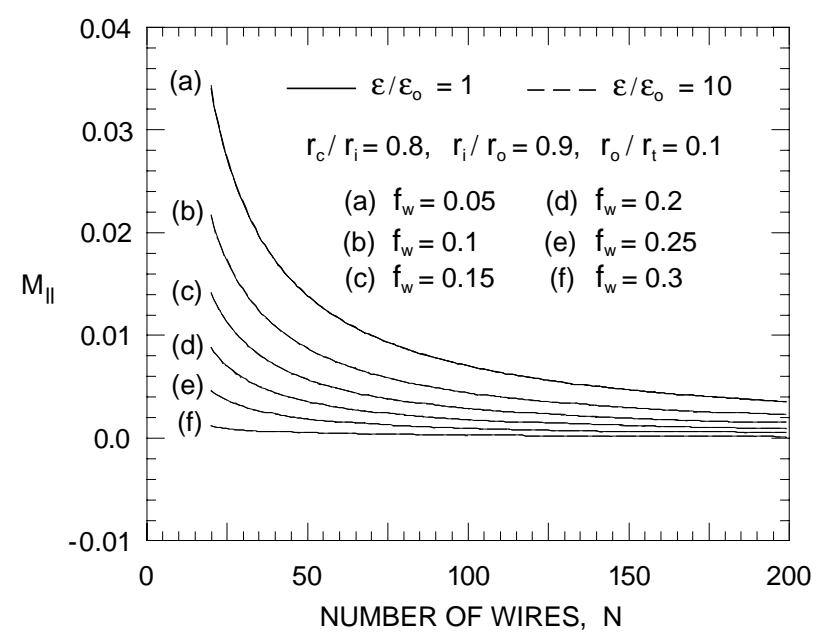

FIG. 5. $M_{\|}$as a function of the total number of wires $N$ for (a) $f_{w}=0.05$, (b) $f_{w}=0.1$, (c) $f_{w}=0.15$, (d) $f_{w}=0.2$, (e) $f_{w}=0.25$, and (f) $f_{w}=0.3$. Here, $M_{\|}$was calculated using Eq. (43) for $r_{c} / r_{i}=0.8, r_{i} / r_{o}=0.9$, and $r_{o} / r_{t}=0.1$.

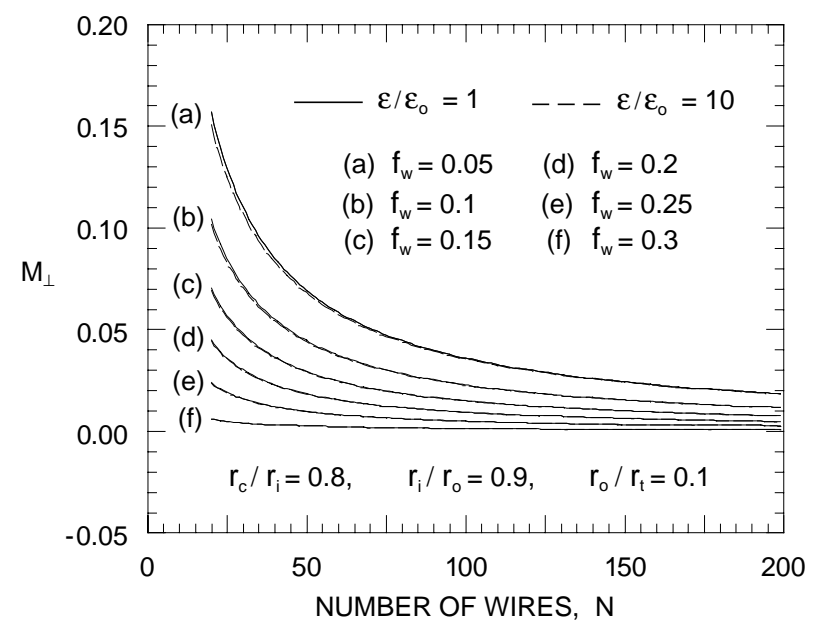

FIG. 6. $M_{\perp}$ as a function of the total number of wires $N$ for (a) $f_{w}=0.05$, (b) $f_{w}=0.1$, (c) $f_{w}=0.15$, (d) $f_{w}=0.2$, (e) $f_{w}=0.25$, and (f) $f_{w}=0.3$. Here, $M_{\perp}$ was calculated using Eq. (44) for $r_{c} / r_{i}=0.8, r_{i} / r_{o}=0.9$, and $r_{o} / r_{t}=0.1$.

useful to present the numerical values of $R_{L}$ and $R_{T}$ as functions of $f_{w}$ and $r_{c} / r_{t}$ so that $M_{\|}$and $M_{\perp}$ can be easily calculated for a given number of wires. Such examples are shown in Figs. 7 and 8, where the values of $R_{L}$ and $R_{T}$ were computed using $M_{\|}$and $M_{\perp}$ obtained from Eqs. (43) and (44) for $N=60$. We can see from the results in Figs. 7 and 8 that the values of $R_{L}$ and $R_{T}$ increase with the value of ratio $r_{c} / r_{t}$ as one can expect from Eqs. (50) and (51). For $\epsilon=\epsilon_{o}$ and $N \geq 30$, we found that the values of $R_{L}$ and $R_{T}$ are practically independent of the number of wires.

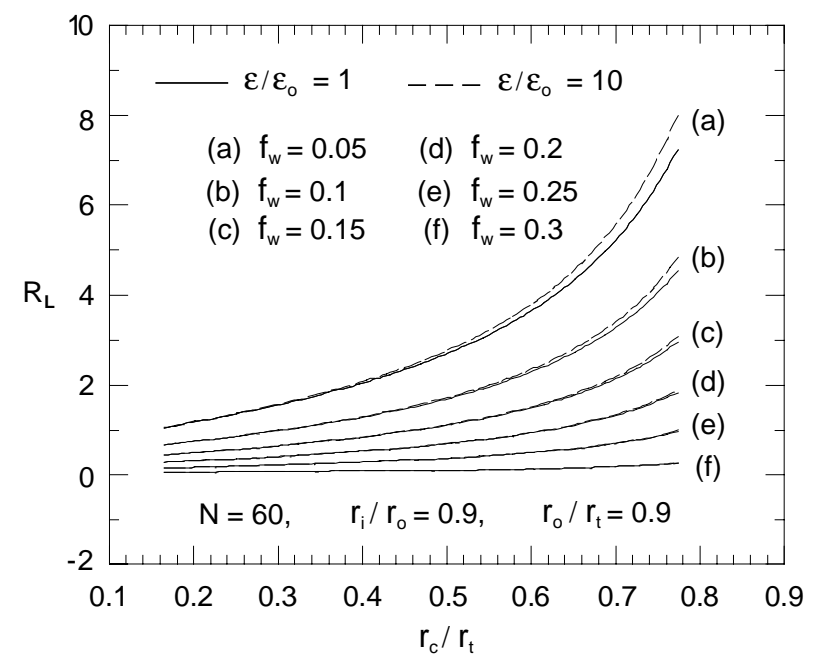

FIG. 7. $R_{L}$ as a function of $r_{c} / r_{t}$ for (a) $f_{w}=0.05$, (b) $f_{w}=$ 0.1 , (c) $f_{w}=0.15$, (d) $f_{w}=0.2$, (e) $f_{w}=0.25$, and (f) $f_{w}=$ 0.3 . Here, $R_{L}$ was calculated using the definition in Eq. (50) for $N=60, r_{i} / r_{o}=0.9$, and $r_{o} / r_{t}=0.9$. 


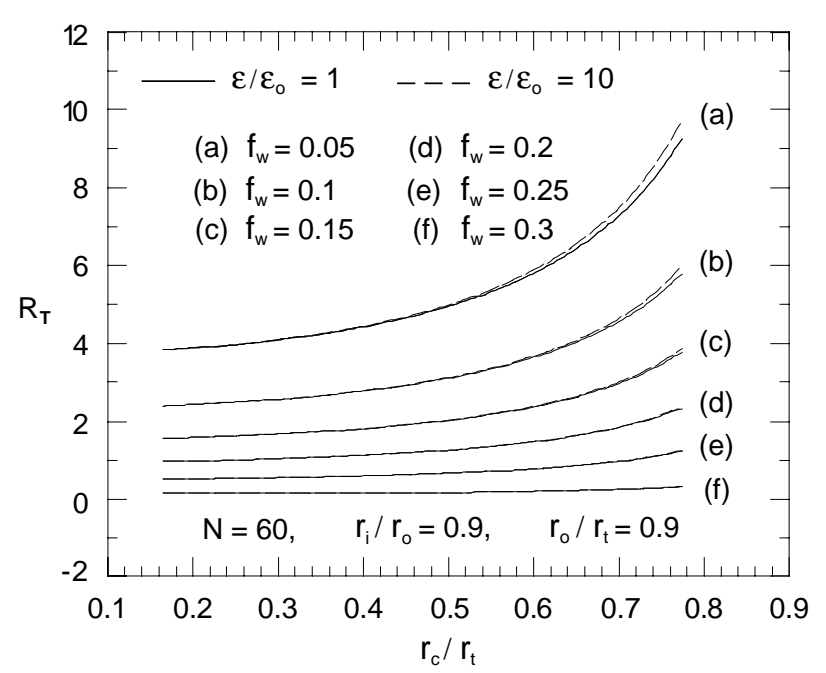

FIG. 8. $\quad R_{T}$ as a function of $r_{c} / r_{t}$ for (a) $f_{w}=0.05$, (b) $f_{w}=$ 0.1 , (c) $f_{w}=0.15$, (d) $f_{w}=0.2$, (e) $f_{w}=0.25$, and (f) $f_{w}=$ 0.3 . Here, $R_{T}$ was calculated using the definition in Eq. (51) for $N=60, r_{i} / r_{o}=0.9$, and $r_{o} / r_{t}=0.9$.

\section{COMPARISON WITH MAFIA COMPUTATIONS}

In the long wavelength regime, $k r_{t} \ll 1$, calculations of the space-charge coupling impedances for homogeneous chambers with an arbitrary cross section can be greatly simplified by reducing the problem to a $2 \mathrm{D}$ electrostatic problem. It was demonstrated in [11] that the value at $r=r_{b}$ of the potential $\psi$, which satisfies $\nabla^{2} \psi=-\delta(r-$ $\left.r_{b}\right) / r_{b}$, gives us directly the so-called space-charge $g$ factor for a hollow thin beam of radius $r_{b}, g_{L}=\psi\left(r_{b}\right)$. The longitudinal space-charge impedance of a hollow beam is related to $g_{L}$ as $Z_{\|}=\operatorname{ing}_{L} Z_{0} /\left(\beta \gamma^{2}\right)$. As defined here,
$g_{L}$ is equal to $\ln \left(r_{t} / r_{b}\right)$ for a smooth circular pipe of radius $r_{t}$ with perfectly conducting walls. The space-charge impedance for the uniform beam, given by Eq. (39), can be rewritten in terms of $g_{L}$ of the hollow beam as $Z_{\|}=$ in $\left(g_{L}+1 / 2\right) Z_{0} /\left(\beta \gamma^{2}\right)$; see Ref. [11] for detail.

Similarly, the transverse space-charge impedance of a homogeneous chamber can be written as $Z_{\perp}=$ $i g_{T} R Z_{0} /\left(\beta^{2} \gamma^{2} r_{b}^{2}\right)$, where $g_{T}$ is the transverse $g$ factor. It is the same for hollow and uniform beams, and equal to $1-\left(r_{b} / r_{t}\right)^{2}$ in a smooth circular pipe of radius $b$ with perfectly conducting walls. The factor $g_{T}$ can be found by solving the following boundary problem in the chamber cross section: $\nabla^{2} \phi=-\cos \theta \delta\left(r-r_{b}\right) / r_{b}$, with trivial boundary conditions. Then, for an axisymmetric case, $g_{T}$ is simply equal to the maximal value $\phi\left(r_{b}\right)$ of the potential in the beam region [11].

From these considerations and Eqs. (41) and (42), one can relate the $g$ factors and the screening coefficients $M_{\|}$ and $M_{\perp}$ :

$$
g_{L}=\left(1-M_{\|}\right) \ln \frac{r_{c}}{r_{b}}+M_{\|} \ln \frac{r_{t}}{r_{b}},
$$

and

$$
g_{T}=\left(1-\frac{r_{b}^{2}}{r_{c}^{2}}\right)\left(1-M_{\perp}\right)+\left(1-\frac{r_{b}^{2}}{r_{t}^{2}}\right) M_{\perp} .
$$

One should emphasize that the space-charge $g$ factors depend on the beam size $r_{b}$, while the coefficients $M_{\|}$and $M_{\perp}$ do not.

For some particular cases the boundary problems presented above have simple analytical solutions. In a general case, one can solve them numerically for an arbitrary geometry of the cross section by means of one

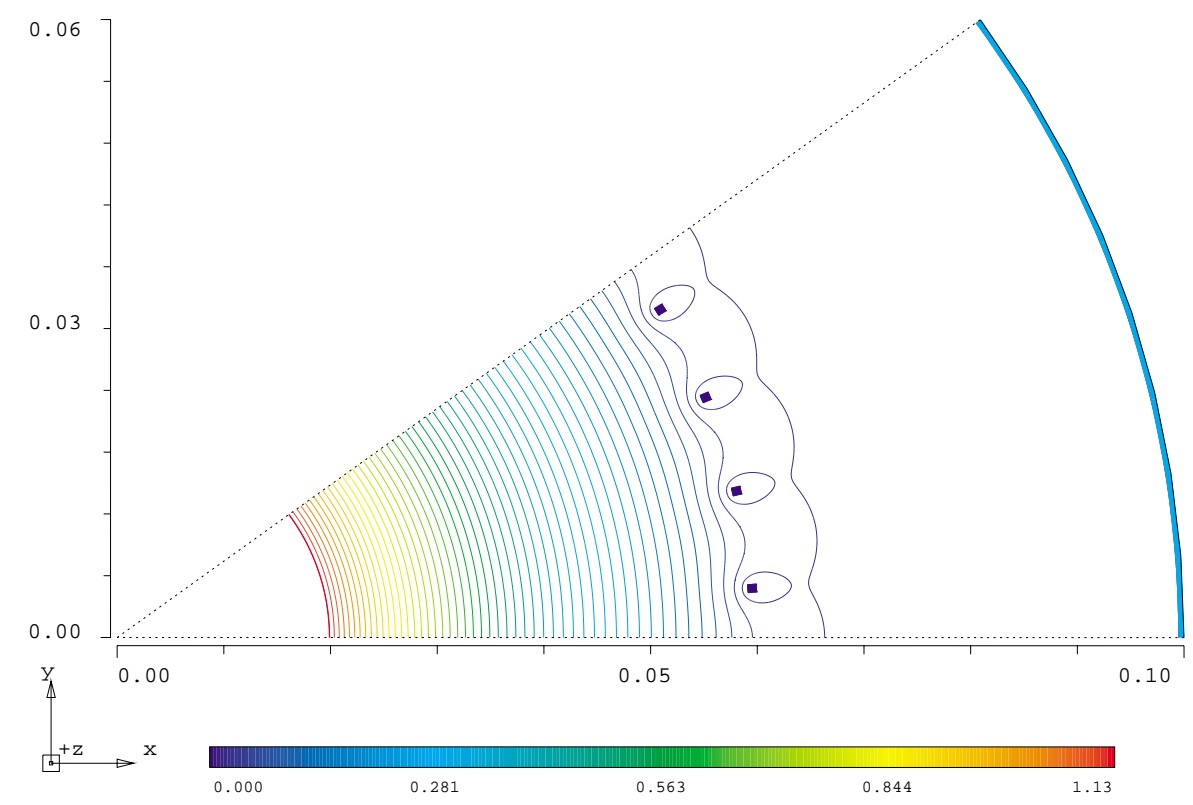

FIG. 9. (Color) Fifty equipotential lines for a hollow beam in the circular chamber with $N=40$ wires. Only one $36^{\circ}$ sector with four wires (blue squares) is shown. The axes show longitudinal $(x)$ and vertical $(y)$ coordinates of the chamber cross section in our MAFIA model in meters. The value of the normalized potential is indicated by the color scale at the bottom. 


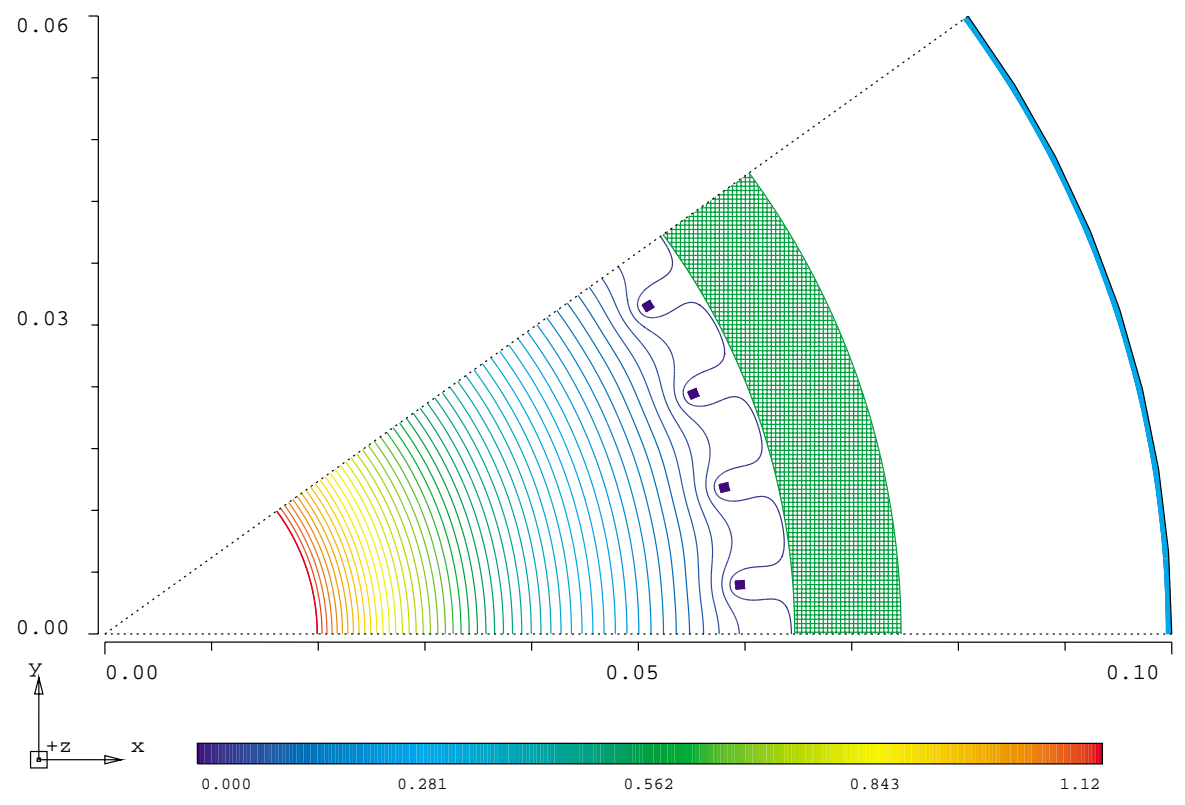

FIG. 10. (Color) The same as in Fig. 9, but with the ceramic layer (green).

of numerous $2 \mathrm{D}$ electrostatic codes. We use the $2 \mathrm{D}$ static solver in the MAFIA code package [14] to calculate the $g$ factors for a circular vacuum chamber with screening wires, with or without an inner ceramic chamber between the screening wire cage and the outer metal chamber wall. In the longitudinal case, for a circular chamber it is enough to solve the problem only in an angular sector containing one wire with periodic boundary conditions. For a large number $N$ of wires, it is more convenient, from the numerical viewpoint, to work with a wider sector containing a few wires, as shown in Fig. 9 for $N=40$, $f_{w}=0.1$, and $r_{c} / r_{t}=0.6$. The sector here is $36^{\circ}$ and contains four wires. We solve for the electric potential $\psi$ produced by a normalized electric charge uniformly

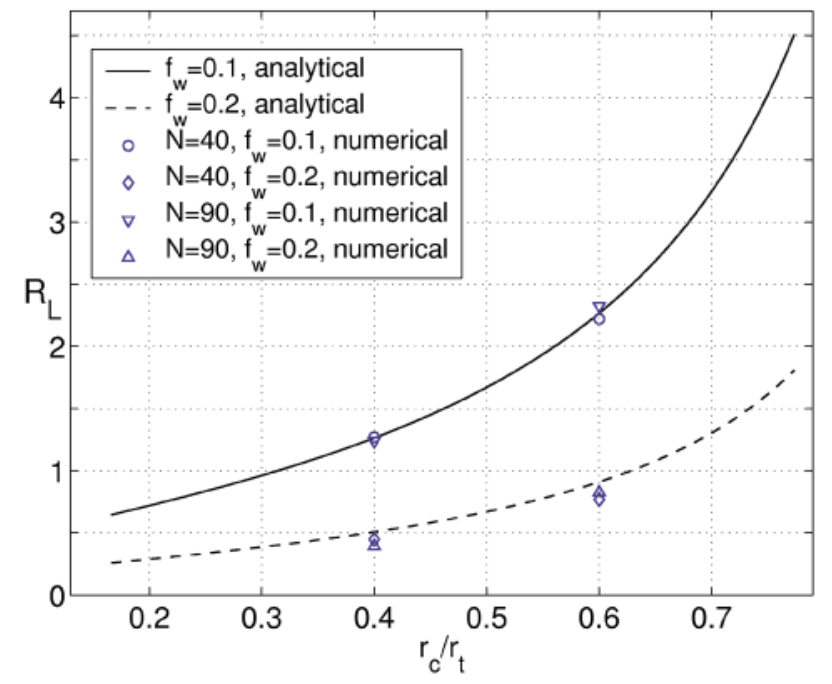

FIG. 11. Comparison of analytical and numerical results for the longitudinal factor $R_{L}$. distributed in (a sector of) a thin hollow beam of radius $r_{b}\left(r_{b} / r_{t}=0.2\right.$ in Figs. 9 and 10). The value of the potential in the beam region gives us the longitudinal factor $g_{L}$. Figure 9 shows 50 equipotential lines, with the maximum in the beam region, and zero on the wall and the wires. One can see that 40 wires screen the beam field rather effectively. Adding a ceramic layer behind the wires (Fig. 10) helps a little, but changes are very small in this case.

One can note in Figs. 9 and 10 that the wires have square cross sections, while the analytical study above was carried out for the circular wires. Our numerical approach is sensitive enough that the results depend on the wire shape, even for thin wires. Without going into much detail (we hope to discuss this topic elsewhere), we just mention that in our MAFIA computations the wires with an equivalent square cross section were used. A square wire with side $s=2[2 E(1 / 2)-K(1 / 2)] r_{w} \approx 1.694 r_{w}$, where $E(x), K(x)$ are the complete elliptic integrals, gives the same screening as a thin wire of radius $r_{w}$. This statement was checked numerically using very dense meshes in

TABLE I. Numerical results for $f_{w}=0.1$.

\begin{tabular}{cccccc}
\hline \hline$r_{c} / r_{t}$ & $N$ & $\epsilon / \epsilon_{0}$ & $g_{L}$ & $M_{\|}$ & $R_{L}$ \\
\hline 0.4 & 40 & 1 & 0.7214 & 0.0308 & 1.270 \\
0.4 & 90 & 1 & 0.7056 & 0.0136 & 1.239 \\
0.4 & $*$ & $\ldots$ & 0.6931 & 0 & $\ldots$ \\
0.6 & 40 & 1 & 1.1255 & 0.0526 & 2.221 \\
0.6 & 40 & 10 & 1.1246 & 0.0509 & 2.146 \\
0.6 & 90 & 1 & 1.1115 & 0.0252 & 2.324 \\
0.6 & $*$ & $\ldots$ & 1.0986 & 0 & $\ldots$ \\
\hline \hline
\end{tabular}




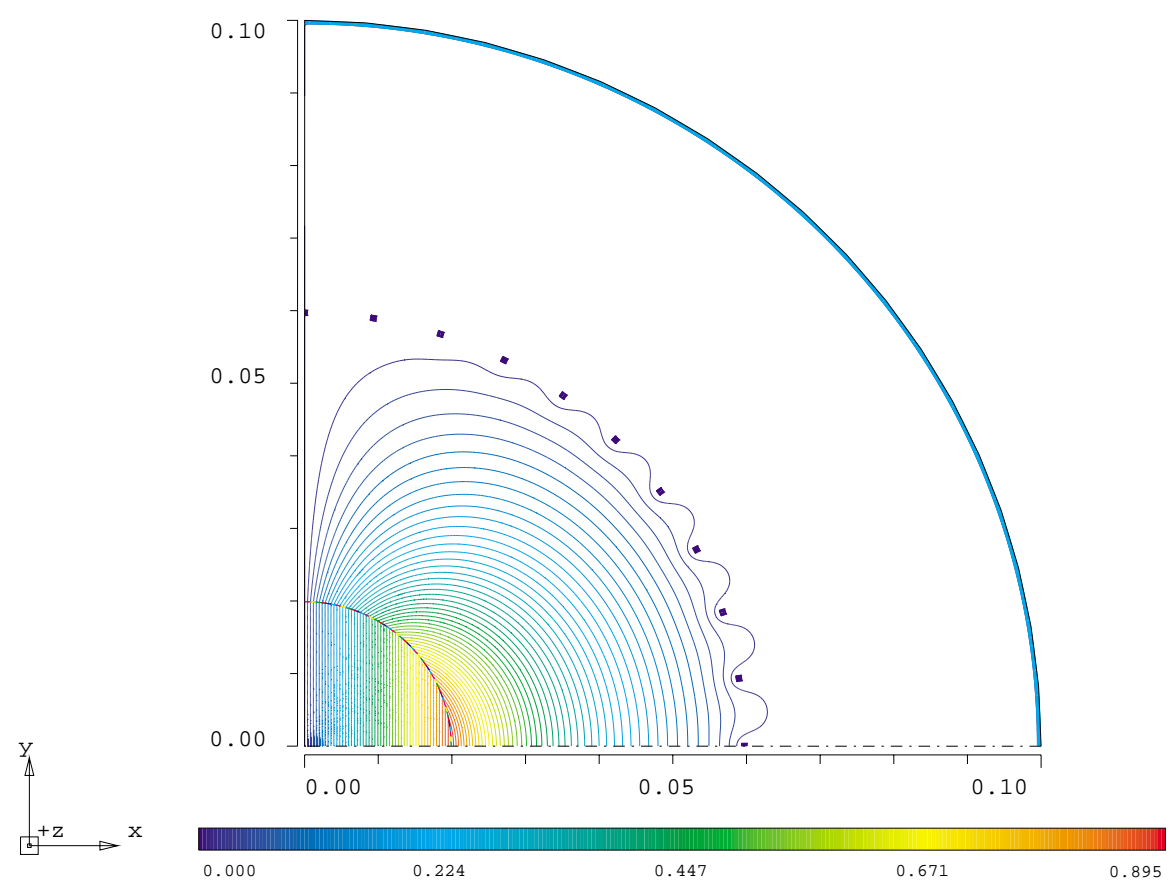

FIG. 12. (Color) Fifty equipotential lines for a hollow beam with a $\cos \theta$ charge distribution in the circular chamber with $N=40$ wires. Only one-quarter of the chamber cross section is shown. The dimensions are in meters. The value of the normalized potential is indicated by the color scale at the bottom.

a couple of cases, and results for the coefficients $M_{\|}$and $M_{\perp}$ agree within a few percent.

The comparison of analytical and MAFIA results for the longitudinal factor $R_{L}$ with $\epsilon / \epsilon_{0}=1$ is presented in Fig. 11. The agreement is excellent for $f_{w}=0.1$, and good for the thicker wires, with $f_{w}=0.2$. The numerical results also confirm the conclusion of the previous section that $R_{L}$ is independent of the number of wires when $N \gg 1$. One should note that in all the numerical cases the space-charge impedance (proportional

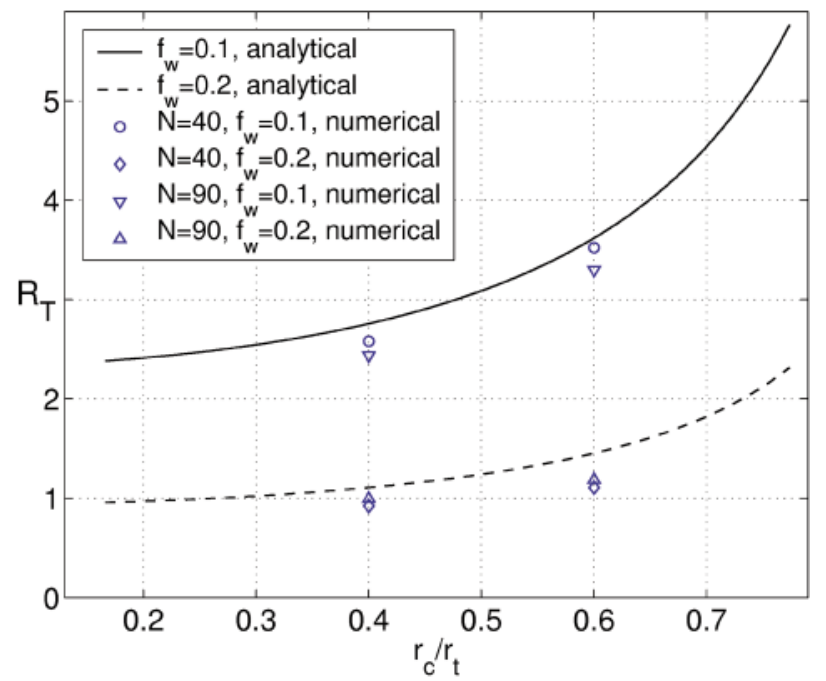

FIG. 13. Comparison of analytical and numerical results for the transverse factor $R_{T}$. to $g_{L}$ ) is very close to that of a continuous metal screen placed at $r=r_{c}$, and depends essentially only on $r_{c} / r_{t}$ as evidenced by Table I for $f_{w}=0.1$ and $r_{b} / r_{t}=0.2$. The continuous screen cases correspond to $f_{w}=1$ and are marked by $*$ in the table. Introducing a dielectric layer as shown in Fig. 10 has very little effect for this layout (compare the fifth and fourth lines in Table I).

For the transverse space-charge impedance computations, we work with one-quarter of the chamber cross section, imposing a symmetric boundary condition at the azimuth $\theta=0$, and an antisymmetric boundary condition at $\theta=\pi / 2$. The dipole charge distribution $\cos \theta$ in a thin hollow beam is approximated by the ring region $r=r_{b}$ split into 45 segments, each carrying a charge proportional to its $\cos \theta$. The solution for the potential is also proportional to $\cos \theta$, and its maximal value at the beam gives the transverse space-charge factor $g_{T}$. Figure 12 shows 50 equipotential lines for the transverse case in the same layout as in Figs. 9 and 10.

The comparison of analytical predictions for the transverse factor $R_{T}$ with the numerical results is presented in Fig. 13. While the agreement is not as good here as for the longitudinal case, with the numerical results systematically below the analytical results, it is still quite reasonable.

\section{CONCLUSIONS}

For a charged-particle beam propagating inside of an rf-shielding cage surrounded by concentric ceramic and conducting beam pipes, the electrostatic fields due to sinusoidal longitudinal and transverse perturbations have been 
calculated analytically for the case in which both the cage and wires have circular cross sections. The rf cage considered is made of evenly spaced conducting wires. Only the dipole mode has been treated for the transverse perturbation. We have assumed that the beam has a uniform charge distribution and the unperturbed system is azimuthally symmetric. Using the calculated fields, we have derived simple formulas shown in Eqs. (41) and (42) for the coupling impedances in the long wavelength region. Numerical examples were given to show the shielding effects of the rf cage. Our results show that, in the long wavelength regime, the space-charge impedance does not have strong dependence on the dielectric constant of the ceramic pipe, and the size of wires is not important when the number of wires is larger than 100. Comparison between analytical estimates and the results produced by the field-solver computer program MAFIA shows good agreement.

\section{ACKNOWLEDGMENTS}

The initial study of this topic was sponsored by the Accelerator Technology (AT) Division of Los Alamos National Laboratory and the AUSTRON Project. T. S. F. W. thanks the AT Division and the AUSTRON Project for the support he received during the course of this study. T. S.F.W. also thanks Dr. Phil Bryant for his encouragement and comments as well as his suggestions. R. L. G. acknowledges support from the Department of Energy. The authors thank Dr. B. Zotter for his comments.

\section{APPENDIX A: DETAILED DERIVATION OF PERTURBED FIELDS (NO CERAMIC PIPE)}

\section{The longitudinal perturbed field}

We first consider the solution of Eq. (1) for the case without wire. We notice that for $r \leq r_{b}$ Eq. (1) admits the solution $\left[\left(\sigma / \epsilon_{o} k^{2}\right)+B I_{0}(k r)\right] e^{i k z}$, where $B$ is a constant to be determined and the unphysical solution $K_{0}(x)$ has been excluded. In the $r_{b} \leq r_{s} \leq r_{t}$ region Eq. (1) has a general solution $\left[A_{1} K_{0}(k r)+A_{2} I_{0}(k r)\right] e^{i k z}$, here $A_{1}$ and $A_{2}$ are constants to be determined. Using the conditions $\Phi\left(r_{t}\right)=0$ and the continuity of electric flux and potential at $r=r_{b}$, we can derive the following equations:

$$
\begin{gathered}
A_{1} K_{0}\left(k r_{t}\right)+A_{2} I_{0}\left(k r_{t}\right)=0, \\
-A_{1} K_{1}\left(k r_{b}\right)+A_{2} I_{1}\left(k r_{b}\right)=B I_{1}\left(k r_{b}\right),
\end{gathered}
$$

and

$$
A_{1} K_{0}\left(k r_{b}\right)+A_{2} I_{0}\left(k r_{b}\right)=\frac{\sigma}{\epsilon_{o} k^{2}}+B I_{0}\left(k r_{b}\right) .
$$

Solving these equations leads to the following solution of Eq. (1):

$$
\Phi=\phi_{b}=b_{\|}\left[K_{0}(k r)-\frac{I_{0}(k r)}{h_{0}\left(k r_{t}\right)}\right] e^{i k z},
$$

for $r_{t} \geq r \geq r_{b}$, and

$$
\begin{aligned}
\Phi & =\phi_{b} \\
& =\left\{\frac{\sigma}{\epsilon_{o} k^{2}}-b_{\|} I_{0}(k r)\left[\frac{1}{h_{0}\left(k r_{t}\right)}+\frac{1}{h_{1}\left(k r_{b}\right)}\right]\right\} e^{i k z},
\end{aligned}
$$

for $r \leq r_{b}$, where

$$
b_{\|}=\left(\frac{\sigma r_{b}}{\epsilon_{o} k}\right) I_{1}\left(k r_{b}\right),
$$

and

$$
h_{n}(x)=I_{n}(x) / K_{n}(x) .
$$

To solve Eq. (1) for the case when wires are included, we first consider the solution in the region of $r_{t} \geq r \geq r_{c}$. In the presence of wires, the beam field will induce electric charges on the surfaces of wires. These induced charges also have an electric potential associated with them. We then assume that the induced charges on each wire create a potential which can be expanded into a Fourier series of $e^{i n \psi}$ as

$$
\phi_{w}=\sum_{n=-\infty}^{\infty}\left[C_{n} K_{n}(k \rho)+D_{n} I_{n}(k \rho)\right] e^{i n \psi} e^{i k z},
$$

where $C_{n}$ and $D_{n}$ are the coefficients to be determined. Note that Eq. (A8) is virtually the multipole expansion of the induced field in the wire coordinate system. Using the addition theorem of Bessel functions [15], $\phi_{w}$ can also be expressed in the global coordinate variables as

$$
\begin{aligned}
\phi_{w}= & \sum_{l=-\infty}^{\infty} \sum_{n=-\infty}^{\infty} I_{n+l}\left(k r_{c}\right) \\
& \times\left[(-1)^{n} C_{n} K_{l}(k r)+D_{n} I_{l}(k r)\right] e^{i l \theta} e^{i k z} .
\end{aligned}
$$

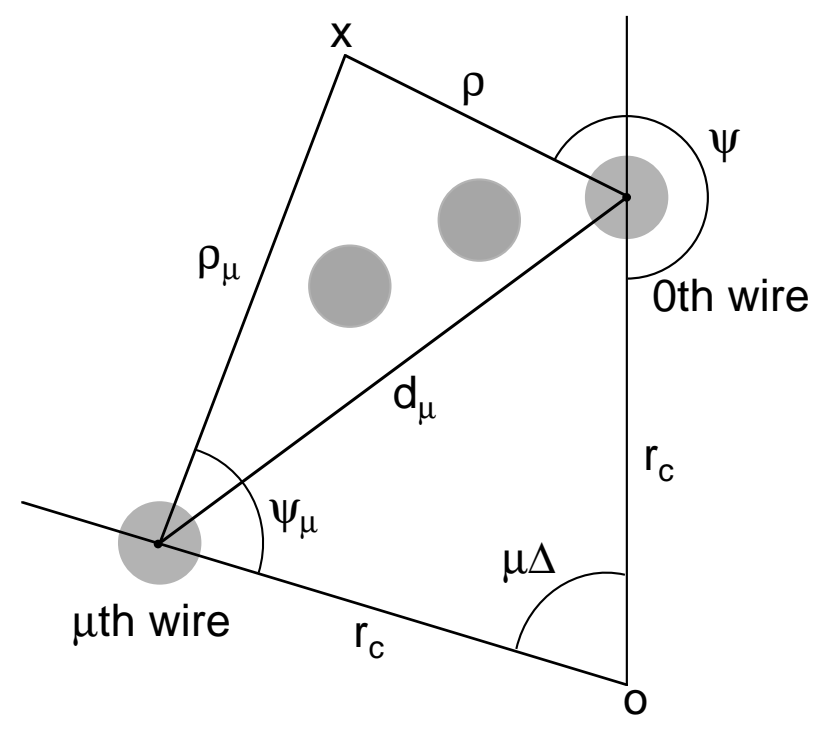

FIG. 14. Shown is the position of a point $x$ represented by the coordinates $(\rho, \psi)$ and $\left(\rho_{\mu}, \psi_{\mu}\right)$ in the local coordinate systems of the 0 th wire and the $\mu$ th wire, respectively. 
Applying the boundary condition of $\phi_{w}=0$ at $r=r_{t}$, we have

$$
D_{n}=-\sum_{l=-\infty}^{\infty} \sum_{j=-\infty}^{\infty}(-1)^{l} C_{l} \frac{I_{n+j}\left(k r_{c}\right) I_{j+l}\left(k r_{c}\right)}{h_{j}\left(k r_{t}\right)}
$$

Since all wires are electrically identical and are evenly distributed, one can study the field around wires by considering the electric potential around any individual wire.
Thus, we call the wire under consideration the 0th wire and number all others by their relative locations with respect to the 0th wire counterclockwise. For an arbitrary point $x$ near the 0th wire, as shown in Fig. 14, we use $(\rho, \psi)$ and $\left(\rho_{\mu}, \psi_{\mu}\right)$ to denote the coordinates of point $x$ in the local coordinate systems of the 0th wire and the $\mu$ th wire, respectively. Let $\Psi_{w}$ be the sum of the induced potentials at point $x$ from all wires except the 0th wire. Then, using Eq. (A8) and the addition theorem of Bessel functions we can derive

$$
\begin{aligned}
\Psi_{w} & =\sum_{\mu=1}^{N-1} \sum_{n=-\infty}^{\infty}\left[C_{n} K_{n}\left(k \rho_{\mu}\right)+D_{n} I_{n}\left(k \rho_{\mu}\right)\right] e^{i n \psi_{\mu}} e^{i k z} \\
& =\sum_{l=-\infty}^{\infty} \sum_{n=-\infty}^{\infty}\left[\sum_{\mu=1}^{N-1} e^{i(n+l)(\pi-\mu \Delta) / 2}\right]\left[C_{l} K_{l-n}\left(k d_{\mu}\right)+D_{l} I_{l-n}\left(k d_{\mu}\right)\right] I_{n}(k \rho) e^{i n \psi} e^{i k z},
\end{aligned}
$$

where $d_{\mu}$ is the distance between the centers of the 0th and the $\mu$ th wire.

The total electric potential around the 0th wire, including the field due to the perturbation in the beam, is

$$
\Phi=\phi_{b}+\phi_{w}+\Psi_{w} .
$$

On the surface of each wire, the potential due to the induced charges should cancel the potential due to the beam and the potential contributed by all other wires, i.e., $\Phi=0$ at $\rho=\rho_{w}$. Thus, substituting Eqs. (A4), (A8), and (A11) into Eq. (A12), we obtain that on the surface of the 0th wire

$$
\sum_{l=-\infty}^{\infty} \sum_{\mu=1}^{N-1} e^{i(n+l)(\pi-\mu \Delta) / 2}\left[C_{l} K_{l-n}\left(k d_{\mu}\right)+D_{l} I_{l-n}\left(k d_{\mu}\right)\right]+\frac{C_{n}}{h_{n}\left(k \rho_{w}\right)}+D_{n}+b_{\|}\left[K_{n}\left(k r_{c}\right)-\frac{I_{n}\left(k r_{c}\right)}{h_{0}\left(k r_{t}\right)}\right]=0 .
$$

Using Eq. (A10) and the relation

$$
\begin{aligned}
\sum_{l=-\infty}^{\infty} \sum_{\mu=1}^{N-1} e^{i(n+l)(\pi-\mu \Delta) / 2} D_{l} I_{l-n}\left(k d_{\mu}\right)= & -\sum_{l=-\infty}^{\infty} \sum_{\mu=1}^{N-1} e^{i(n+l)(\pi-\mu \Delta) / 2} \\
& \times \sum_{m=-\infty}^{\infty}(-1)^{m} C_{m} \sum_{j=-\infty}^{\infty} \frac{I_{m+j}\left(k r_{c}\right) I_{j+l}\left(k r_{c}\right)}{h_{j}\left(k r_{t}\right)} I_{l-n}\left(k d_{\mu}\right) \\
= & -\sum_{m=-\infty}^{\infty}(-1)^{m} C_{m} \sum_{\mu=1}^{N-1} e^{i n(\pi-\mu \Delta)} \sum_{j=-\infty}^{\infty} \frac{I_{m+j}\left(k r_{c}\right)}{h_{j}\left(k r_{t}\right)} \\
& \times \sum_{l=-\infty}^{\infty} e^{i(l-n)(\pi-\mu \Delta) / 2} I_{j+l}\left(k r_{c}\right) I_{l-n}\left(k d_{\mu}\right) \\
= & (-1)^{n+1} \sum_{m=-\infty}^{\infty}(-1)^{m} C_{m} \sum_{j=-\infty}^{\infty} \frac{I_{m+j}\left(k r_{c}\right) I_{j+n}\left(k r_{c}\right)}{h_{j}\left(k r_{t}\right)} \sum_{\mu=1}^{N-1} e^{i j \mu \Delta},
\end{aligned}
$$

we derive from Eq. (A13):

$$
\begin{gathered}
\sum_{l=-\infty}^{\infty}\left[\sum_{\mu=1}^{N-1} e^{i(n+l)(\pi-\mu \Delta) / 2} K_{l-n}\left(k d_{\mu}\right)\right] C_{l}+\frac{C_{n}}{h_{n}\left(k \rho_{w}\right)}- \\
\sum_{l=-\infty}^{\infty}\left\{\sum_{j=-\infty}^{\infty} \frac{I_{n+j}\left(k r_{c}\right) I_{j+l}\left(k r_{c}\right)}{h_{j}\left(k r_{t}\right)}\left[1+(-1)^{n} \sum_{\mu=1}^{N-1} e^{i j \mu \Delta}\right]\right\}(-1)^{l} C_{l}+b_{\|}\left[K_{n}\left(k r_{c}\right)-\frac{I_{n}\left(k r_{c}\right)}{h_{0}\left(k r_{t}\right)}\right]=0,
\end{gathered}
$$

where $C_{k}$ is the unknown to be solved for.

The closed-form solution of Eq. (A15) is difficult, if not impossible, to obtain. However, in the regime of $k \rho_{w} \ll$ $k r_{c}<k r_{t} \ll 1$, one can expand the solution in the power series of $h_{n}\left(k \rho_{w}\right)$. If the multipole coupling is neglected by retaining only the $l=n$ terms in Eq. (A15), the lowest order solution is 


$$
C_{n} \approx-\frac{b_{\|} h_{n}\left(k \rho_{w}\right)}{G_{\| n}}\left[K_{n}\left(k r_{c}\right)-\frac{I_{n}\left(k r_{c}\right)}{h_{0}\left(k r_{t}\right)}\right]
$$

where

$$
G_{\| n}=1+(-1)^{n} h_{n}\left(k \rho_{w}\right) \sum_{\mu=1}^{N-1} e^{-i n \mu \Delta} K_{0}\left(k d_{\mu}\right)-h_{n}\left(k \rho_{w}\right) \sum_{j=-\infty}^{\infty} \frac{\left[I_{n+j}\left(k r_{c}\right)\right]^{2}}{h_{j}\left(k r_{t}\right)}\left[1+(-1)^{n} \sum_{\mu=1}^{N-1} e^{i j \mu \Delta}\right] .
$$

The contribution of the potential from all wires can then be expressed in terms of global coordinate variables as

$$
\phi_{w}+\Psi_{w}=N \sum_{p=-\infty}^{\infty} \sum_{n=-\infty}^{\infty}(-1)^{n} C_{n} I_{n+p N}\left(k r_{c}\right)\left[K_{p N}(k r)-\frac{I_{p N}(k r)}{h_{p N}\left(k r_{t}\right)}\right] e^{i p N \theta} e^{i k z},
$$

with $C_{n}$ given in Eq. (A16). In arriving at Eq. (A18), we made use of the relation

$$
\begin{aligned}
\sum_{n=-\infty}^{\infty} D_{n} I_{n+p N}\left(k r_{c}\right) & =-\sum_{n=-\infty}^{\infty} \sum_{l=-\infty}^{\infty}(-1)^{l} C_{l} \sum_{j=-\infty}^{\infty} \frac{I_{n+j}\left(k r_{c}\right) I_{j+l}\left(k r_{c}\right)}{h_{j}\left(k r_{t}\right)} I_{n+p N}\left(k r_{c}\right) \\
& =-\sum_{l=-\infty}^{\infty}(-1)^{l} C_{l} \frac{I_{l+p N}\left(k r_{c}\right)}{h_{p N}\left(k r_{t}\right)}
\end{aligned}
$$

and the equality (holds good only when $\Delta=2 \pi / N$ )

$$
\sum_{j=0}^{N-1} e^{i j l \Delta}= \begin{cases}N, & \text { for } l=p N, p=0, \pm 1, \pm 2, \ldots, \\ 0, & \text { for } l \neq p N\end{cases}
$$

Substituting the potentials in Eqs. (A2) and (A18) into Eq. (A12) yields the following total potential in the region of $r_{i} \geq r \geq r_{c}$ :

$$
\Phi \approx b_{\|}\left[K_{0}(k r)-\frac{I_{0}\left(k r_{c}\right)}{h_{0}\left(k r_{t}\right)}\right] e^{i k z}+N \sum_{p=-\infty}^{\infty} \sum_{n=-\infty}^{\infty}(-1)^{n} C_{n} I_{n+p N}\left(k r_{c}\right)\left[K_{p N}(k r)-\frac{I_{p N}(k r)}{h_{p N}\left(k r_{t}\right)}\right] e^{i p N \theta} e^{i k z} .
$$

Next, we consider the solution in the region of $r \leq r_{c}$. Applying the addition theorem of Bessel functions to Eq. (A8) renders

$$
\phi_{w}=\sum_{l=-\infty}^{\infty} \sum_{n=-\infty}^{\infty}\left[C_{n} K_{n+l}\left(k r_{c}\right)+D_{n} I_{n+l}\left(k r_{c}\right)\right] I_{l}(k r) e^{i l \theta} e^{i k z}
$$

With the aid of Eqs. (A19) and (A20), the contribution of the potential from all wires can be evaluated as

$$
\phi_{w}+\Psi_{w}=N \sum_{p=-\infty}^{\infty} \sum_{n=-\infty}^{\infty} C_{n}\left[K_{n+p N}\left(k r_{c}\right)-(-1)^{n} \frac{I_{n+p N}\left(k r_{c}\right)}{h_{p N}\left(k r_{t}\right)}\right] I_{p N}(k r) e^{i p N \theta} e^{i k z} .
$$

Using the solution for $\phi_{b}$ in Eqs. (A4) and (A5), we derive that

$$
\Phi \approx b_{\|}\left[K_{0}(k r)-\frac{I_{0}(k r)}{h_{0}\left(k r_{t}\right)}\right] e^{i k z}+N \sum_{p=-\infty}^{\infty} \sum_{n=-\infty}^{\infty} C_{n}\left[K_{n+p N}\left(k r_{c}\right)-(-1)^{n} \frac{I_{n+p N}\left(k r_{c}\right)}{h_{p N}\left(k r_{t}\right)}\right] I_{p N}(k r) e^{i p N \theta} e^{i k z},
$$

for $r_{c} \geq r \geq r_{b}$, and

$$
\begin{aligned}
\Phi \approx & \left\{\frac{\sigma}{\epsilon_{o} k^{2}}-I_{0}(k r) b_{\|}\left[\frac{1}{h_{0}\left(k r_{t}\right)}+\frac{1}{h_{1}\left(k r_{b}\right)}\right]\right\} e^{i k z} \\
& +N \sum_{p=-\infty}^{\infty} \sum_{n=-\infty}^{\infty} C_{n}\left[K_{n+p N}\left(k r_{c}\right)-(-1)^{n} \frac{I_{n+p N}\left(k r_{c}\right)}{h_{p N}\left(k r_{t}\right)}\right] I_{p N}(k r) e^{i p N \theta} e^{i k z},
\end{aligned}
$$

for $r \leq r_{b}$. Thus, at $r=0$ we have

$$
\begin{aligned}
\left.E_{z}\right|_{r=0} & =-\left.\frac{\partial \Phi}{\partial z}\right|_{r=0} \\
& \approx-i k N \sum_{n=-\infty}^{\infty} C_{n}\left[K_{n}\left(k r_{c}\right)-(-1)^{n} \frac{I_{n}\left(k r_{c}\right)}{h_{0}\left(k r_{t}\right)}\right] e^{i k z}-\frac{i \sigma}{\epsilon_{o} k}\left\{1-k r_{b}\left[\frac{I_{1}\left(k r_{b}\right)}{h_{0}\left(k r_{t}\right)}+K_{1}\left(k r_{b}\right)\right]\right\} e^{i k z} .
\end{aligned}
$$


If we further take the approximation by considering $n=0$ only, we obtain

$$
\left.E_{z}\right|_{r=0} \approx-i k N C_{0}\left[K_{0}\left(k r_{c}\right)-\frac{I_{0}\left(k r_{c}\right)}{h_{0}\left(k r_{t}\right)}\right] e^{i k z}-\frac{i \sigma}{\epsilon_{o} k}\left\{1-k r_{b}\left[\frac{I_{1}\left(k r_{b}\right)}{h_{0}\left(k r_{t}\right)}+K_{1}\left(k r_{b}\right)\right]\right\} e^{i k z} .
$$

Now, from Eq. (A17),

$$
\begin{aligned}
& G_{\| 0}=1+h_{0}\left(k \rho_{w}\right) \sum_{\mu=1}^{N-1} K_{0}\left(k d_{\mu}\right)-h_{0}\left(k \rho_{w}\right) \sum_{j=-\infty}^{\infty} \frac{\left[I_{j}\left(k r_{c}\right)\right]^{2}}{h_{j}\left(k r_{t}\right)}\left[1+\sum_{\mu=1}^{N-1} e^{i j \mu \Delta}\right] \\
&=1+h_{0}\left(k \rho_{w}\right)\left\{\sum_{l=-\infty}^{\infty} K_{l}\left(k r_{c}\right) I_{l}\left(k r_{c}\right) \sum_{\mu=1}^{N-1} e^{i l \mu \Delta}-N \sum_{p=-\infty}^{\infty} \frac{\left[I_{p N}\left(k r_{c}\right)\right]^{2}}{h_{p N}\left(k r_{t}\right)}\right\} \\
&=1+h_{0}\left(k \rho_{w}\right)\left\{(N-1) K_{0}\left(k r_{c}\right) I_{0}\left(k r_{c}\right)-N \sum_{p=-\infty}^{\infty} \frac{\left[I_{p N}\left(k r_{c}\right)\right]^{2}}{h_{p N}\left(k r_{t}\right)}-2 \sum_{q=1}^{N-1} K_{q}\left(k r_{c}\right) I_{q}\left(k r_{c}\right)\right. \\
&\left.+2 \sum_{p=1}^{\infty}\left[(N-1) K_{p N}\left(k r_{c}\right) I_{p N}\left(k r_{c}\right)-\sum_{q=1}^{N-1} K_{q+p N}\left(k r_{c}\right) I_{q+p N}\left(k r_{c}\right)\right]\right\} .
\end{aligned}
$$

One can show, by using the small argument expansions of Bessel functions, that for $N \gg 1$ and $k r_{c} \ll 1$,

$$
\begin{gathered}
(N-1) K_{0}\left(k r_{c}\right) I_{0}\left(k r_{c}\right)-2 \sum_{q=1}^{N-1} K_{q}\left(k r_{c}\right) I_{q}\left(k r_{c}\right)+2 \sum_{p=1}^{\infty}\left[(N-1) K_{p N}\left(k r_{c}\right) I_{p N}\left(k r_{c}\right)-\sum_{q=1}^{N-1} K_{q+p N}\left(k r_{c}\right) I_{q+p N}\left(k r_{c}\right)\right] \\
\approx-(N-1) \ln \left(k r_{c}\right)-\sum_{q=1}^{N-1} \frac{1}{q}+\sum_{p=1}^{\infty}\left[\frac{N-1}{p N}-\sum_{q=1}^{N-1} \frac{1}{p N+q}\right] \\
\approx-(N-1) \ln \left(k r_{c}\right)-\ln N
\end{gathered}
$$

and

$$
\sum_{p=-\infty}^{\infty} \frac{\left[I_{p N}\left(k r_{c}\right)\right]^{2}}{h_{p N}\left(k r_{t}\right)}=\sum_{p=-\infty}^{\infty}\left[\frac{I_{p N}\left(k r_{c}\right)}{I_{p N}\left(k r_{t}\right)}\right]^{2} K_{p N}\left(k r_{t}\right) I_{p N}\left(k r_{t}\right) \approx-\ln \left(k r_{t}\right)-\frac{1}{N} \ln \left[1-\left(\frac{r_{c}}{r_{t}}\right)^{2 N}\right] .
$$

Substituting Eqs. (A29) and (A30) into Eq. (A28) yields

$$
G_{\| 0} \approx 1-h_{0}\left(k \rho_{w}\right)\left[(N-1) \ln \left(k r_{c}\right)+\ln N\right]+h_{0}\left(k \rho_{w}\right)\left\{N \ln \left(k r_{t}\right)+\ln \left[1-\left(\frac{r_{c}}{r_{t}}\right)^{2 N}\right]\right\} .
$$

Letting $n=0$ in Eq. (A16) and using Eq. (A31), we obtain

$$
C_{0} \approx \frac{b_{\|} \ln \left(r_{c} / r_{t}\right)}{N \ln \left(r_{t} / r_{c}\right)-\ln \left(\pi f_{w}\right)+\ln \left[1-\left(r_{c} / r_{t}\right)^{2 N}\right]},
$$

where $f_{w}$ is the wire filling factor defined in Eq. (22). Substituting Eq. (A32) into Eq. (A27) and applying the small argument expansions of Bessel functions, we derive

$$
\left.E_{z}\right|_{r=0} \approx \frac{-i \sigma k r_{b}^{2}}{4 \epsilon_{o}}\left\{\left[1+2 \ln \left(\frac{r_{c}}{r_{b}}\right)\right]\left(1-M_{\|}\right)+\left[1+2 \ln \left(\frac{r_{t}}{r_{b}}\right)\right] M_{\|}\right\},
$$

where $M_{\|}$is given in Eq. (47) in the main text.

\section{The transverse perturbed field}

For the case of transverse perturbation, the Poisson equation we want to solve is Eq. (27). Because the system under consideration is invariant under the transformation $\theta \rightarrow-\theta$, and because the Bessel functions that will appear in the solution have the properties of $I_{-n}(x)=I_{n}(x)$ and $K_{-n}(x)=K_{n}(x)$, we will first solve the equation

$$
\frac{1}{r} \frac{\partial}{\partial r}\left(r \frac{\partial \Phi}{\partial r}\right)+\frac{1}{r^{2}} \frac{\partial^{2} \Phi}{\partial \theta^{2}}+\frac{\partial^{2} \Phi}{\partial z^{2}}=-\frac{\sigma \bar{d}}{2 \epsilon_{o}} \delta\left(r-r_{b}\right) e^{i \theta} e^{i k z}
$$

The solution of Eq. (27) then can be obtained from the solution of Eq. (A34) by the substitution of $e^{i \theta} / 2 \rightarrow \cos \theta$ appropriately. Following the same procedures as in deriving Eqs. (A4) and (A5), we find that the solution of Eq. (A34) in the absence of wires is 


$$
\phi_{b}=b_{\perp}\left[K_{1}(k r)-\frac{I_{1}(k r)}{h_{1}\left(k r_{t}\right)}\right] e^{i \theta} e^{i k z}
$$

for $r_{t} \geq r \geq r_{b}$, and

for $r \leq r_{b}$, where

$$
\phi_{b}=b_{\perp}\left[\frac{1}{h_{1}\left(k r_{b}\right)}-\frac{1}{h_{1}\left(k r_{t}\right)}\right] I_{1}(k r) e^{i \theta} e^{i k z}
$$

$$
b_{\perp}=\left(\frac{\sigma \bar{d} r_{b}}{2 \epsilon_{o}}\right) I_{1}\left(k r_{b}\right) .
$$

To include the effects due to the rf-shielding wires, we again consider first the region of $r \geq r_{c}$. Different from the case of longitudinal perturbation, the axial symmetry does not exist here, and the angular positions of wires need to be taken into account when describing the potential around each wire. Take the wire located at the angle of $m \Delta$, the $m$ th wire, as an example. When applying the addition theorem of Bessel functions, one has to make a change of variable from $\theta$ to $\theta-m \Delta$ for translating the description of the potential from the global coordinate to the local coordinate around the $m$ th wire. Therefore, the multipole expansion coefficients of the field induced on each wire depend on the location of the wire.

The analysis here will follow the same path as in solving the field for the longitudinal perturbation. Thus, we assume the $m$ th wire induces a potential which can be described in terms of the wire coordinate system variables as

$$
\phi_{w, m}=\sum_{n=-\infty}^{\infty}\left[P_{m, n} K_{n}(k \rho)+Q_{m, n} I_{n}(k \rho)\right] e^{i n \psi} e^{i k z},
$$

where $P_{m, n}$ and $Q_{m, n}$ are the unknowns to be solved for. The contribution from all other $N-1$ wires, $\Psi_{w, m}^{\prime}$, is

$$
\Psi_{w, m}^{\prime}=\sum_{l=-\infty}^{\infty} \sum_{n=-\infty}^{\infty} \sum_{j=0}^{N-1}\left(1-\delta_{j, m}\right) e^{i(n+l)[\pi-(j-m) \Delta] / 2}\left[P_{j, l} K_{l-n}\left(k d_{j, m}\right)+Q_{j, l} I_{l-n}\left(k d_{j, m}\right)\right] I_{n}(k \rho) e^{i n \psi} e^{i k z},
$$

where $d_{j, m}$ is the distance between the centers of the $m$ th and the $j$ th wires. In the global coordinate system, we have

$$
\phi_{w, m}=\sum_{n=-\infty}^{\infty} \sum_{l=-\infty}^{\infty} I_{l+n}\left(k r_{c}\right)\left[(-1)^{n} P_{m, n} K_{l}(k r)+Q_{m, n} I_{l}(k r)\right] e^{i l \theta} e^{i(l-1) m \Delta} e^{i k z} .
$$

Then, the requirement that the total potential is zero on the surface of the $m$ th wire leads to

$$
\begin{gathered}
\frac{P_{m, n}}{h_{n}\left(k \rho_{w}\right)}+Q_{m, n}+b_{\perp}\left[K_{n+1}\left(k r_{c}\right)-\frac{I_{n+1}\left(k r_{c}\right)}{h_{1}\left(k r_{t}\right)}\right] e^{i m \Delta}+ \\
\sum_{l=-\infty}^{\infty} \sum_{j=0}^{N-1}\left(1-\delta_{j, m}\right) e^{i(n+l)[\pi-(j-m) \Delta] / 2}\left[P_{j, l} K_{l-n}\left(k d_{j, m}\right)+Q_{j, l} I_{l-n}\left(k d_{j, m}\right)\right]=0 .
\end{gathered}
$$

For $m=0$, we have

$$
\begin{gathered}
\frac{P_{n}}{h_{n}\left(k \rho_{w}\right)}+Q_{n}+b_{\perp}\left[K_{n+1}\left(k r_{c}\right)-\frac{I_{n+1}\left(k r_{c}\right)}{h_{1}\left(k r_{t}\right)}\right]+ \\
\sum_{l=-\infty}^{\infty} \sum_{j=0}^{N-1}\left(1-\delta_{j, 0}\right) e^{i(n+l)(\pi-j \Delta) / 2}\left[P_{j, l} K_{l-n}\left(k d_{j}\right)+Q_{j, l} I_{l-n}\left(k d_{j}\right)\right]=0,
\end{gathered}
$$

where $P_{n}=P_{0, n}, Q_{n}=Q_{0, n}$, and $d_{j}=d_{j, 0}$.

Changing index in Eq. (A41) by letting $j=m+q$, one has

$$
\begin{gathered}
\frac{P_{m, n} e^{-i m \Delta}}{h_{n}\left(k \rho_{w}\right)}+Q_{m, n} e^{-i m \Delta}+b_{\perp}\left[K_{n+1}\left(k r_{c}\right)-\frac{I_{n+1}\left(k r_{c}\right)}{h_{1}\left(k r_{t}\right)}\right]+ \\
\sum_{l=-\infty}^{\infty} \sum_{q=0}^{N-1}\left(1-\delta_{q, 0}\right) e^{i(n+l)(\pi-q \Delta) / 2} e^{-i m \Delta}\left[P_{m+q, l} K_{l-n}\left(k d_{q, 0}\right)+Q_{m+q, l} I_{l-n}\left(k d_{q, 0}\right)\right]=0 .
\end{gathered}
$$

It can be seen now that Eqs. (A42) and (A43) have the same form, or $P_{n}$ and $P_{m, n} e^{-i m \Delta}$ have the same solution. We then infer that 


$$
\begin{gathered}
P_{m, n} e^{-i m \Delta}=P_{n}, \\
P_{m+k, n} e^{-i k \Delta}=P_{n} e^{-i m \Delta}=P_{m, n}, \\
Q_{m, n} e^{-i m \Delta}=Q_{n},
\end{gathered}
$$

and

$$
Q_{m+k, n} e^{-i k \Delta}=Q_{n} e^{-i m \Delta}=Q_{m, n} .
$$

Applying these identities to Eq. (A41), the requirement of zero total potential on the surfaces of all wires can be reduced to a single equation similar to Eq. (A15):

$\sum_{l=-\infty}^{\infty} \sum_{\mu=1}^{N-1} e^{i(n+l)(\pi-\mu \Delta) / 2} e^{i \mu \Delta}\left[P_{l} K_{l-n}\left(k d_{\mu}\right)+Q_{l} I_{l-n}\left(k d_{\mu}\right)\right]+\frac{P_{n}}{h_{n}\left(k \rho_{w}\right)}+Q_{n}+b_{\perp}\left[K_{n+1}\left(k r_{c}\right)-\frac{I_{n+1}\left(k r_{c}\right)}{h_{1}\left(k r_{t}\right)}\right]=0$.

Requiring $\phi_{w, m}=0$ at $r=r_{t}$, we can derive from Eq. (A40)

$$
Q_{m, j}=-\sum_{n=-\infty}^{\infty}(-1)^{n} P_{m, n} \sum_{l=-\infty}^{\infty} \frac{I_{l+n}\left(k r_{c}\right) I_{l+j}\left(k r_{c}\right)}{h_{l}\left(k r_{t}\right)},
$$

so that

$$
Q_{j}=-\sum_{n=-\infty}^{\infty}(-1)^{n} P_{n} \sum_{l=-\infty}^{\infty} \frac{I_{l+n}\left(k r_{c}\right) I_{l+j}\left(k r_{c}\right)}{h_{l}\left(k r_{t}\right)}
$$

and

$$
\sum_{l=-\infty}^{\infty} \sum_{\mu=1}^{N-1} e^{i(n+l)(\pi-\mu \Delta) / 2} e^{i \mu \Delta} I_{l-n}\left(k d_{\mu}\right) Q_{l}=(-1)^{n+1} \sum_{m=-\infty}^{\infty}(-1)^{m} P_{m} \sum_{j=-\infty}^{\infty} I_{j+m}\left(k r_{c}\right) \frac{I_{j+n}\left(k r_{c}\right)}{h_{j}\left(k r_{t}\right)} \sum_{\mu=1}^{N-1} e^{i(j+1) \mu \Delta,}
$$

where the addition theorem of Bessel functions has been invoked. Substituting Eq. (A50) into Eq. (A48) and using Eq. (A51) renders

$$
\begin{gathered}
\sum_{l=-\infty}^{\infty} \sum_{\mu=1}^{N-1} e^{i(n+l)(\pi-\mu \Delta) / 2} e^{i \mu \Delta} K_{l-n}\left(k d_{\mu}\right) P_{l}+\frac{P_{n}}{h_{n}\left(k \rho_{w}\right)}- \\
\sum_{l=-\infty}^{\infty} \sum_{j=-\infty}^{\infty}(-1)^{l} \frac{I_{j+l}\left(k r_{c}\right) I_{j+n}\left(k r_{c}\right)}{h_{j}\left(k r_{t}\right)}\left[1+(-1)^{n} \sum_{\mu=1}^{N-1} e^{i(j+1) \mu \Delta}\right] P_{l}+b_{\perp}\left[K_{n+1}\left(k r_{c}\right)-\frac{I_{n+1}\left(k r_{c}\right)}{h_{1}\left(k r_{t}\right)}\right]=0 .
\end{gathered}
$$

Neglecting the multipole coupling by retaining only the $l=n$ terms in Eq. (A52) yields

$$
\begin{aligned}
P_{n}\left[1+(-1)^{n} h_{n}\left(k \rho_{w}\right) \sum_{\mu=1}^{N-1} e^{-i(n-1) \mu \Delta} K_{0}\left(k d_{\mu}\right)\right] & -(-1)^{n} P_{n} h_{n}\left(k \rho_{w}\right) \sum_{j=-\infty}^{\infty} \frac{\left[I_{j+n}\left(k r_{c}\right)\right]^{2}}{h_{j}\left(k r_{t}\right)}\left[1+(-1)^{n} \sum_{\mu=1}^{N-1} e^{i(j+1) \mu \Delta}\right] \\
& =-b_{\perp} h_{n}\left(k \rho_{w}\right)\left[K_{n+1}\left(k r_{c}\right)-\frac{I_{n+1}\left(k r_{c}\right)}{h_{1}\left(k r_{t}\right)}\right] .
\end{aligned}
$$

Equation (A53) has the approximate solution

$$
P_{n} \approx-b_{\perp} h_{n}\left(k \rho_{w}\right)\left[K_{n+1}\left(k r_{c}\right)-\frac{I_{n+1}\left(k r_{c}\right)}{h_{1}\left(k r_{t}\right)}\right] G_{\perp n}^{-1},
$$

where

$$
G_{\perp n}=1+(-1)^{n} h_{n}\left(k \rho_{w}\right)\left\{\sum_{\mu=1}^{N-1} e^{-i(n-1) \mu \Delta} K_{0}\left(k d_{\mu}\right)-\sum_{j=-\infty}^{\infty} \frac{\left[I_{j+n}\left(k r_{c}\right)\right]^{2}}{h_{j}\left(k r_{t}\right)}\left[1+(-1)^{n} \sum_{\mu=1}^{N-1} e^{i(j+1) \mu \Delta}\right]\right\} .
$$


Now, using Eqs. (A40), (A20), and (A50), we can evaluate the potential due to all wires as

$$
\begin{aligned}
\phi_{w, m}+\Psi_{w, m}^{\prime} & =N \sum_{n=-\infty}^{\infty} \sum_{p=-\infty}^{\infty} I_{n+p N+1}\left(k r_{c}\right)\left[(-1)^{n} P_{n} K_{p N+1}(k r)+Q_{n} I_{p N+1}(k r)\right] e^{i(p N+1) \theta} e^{i k z} \\
& =2 N \sum_{p=-\infty}^{\infty} \sum_{n=-\infty}^{\infty} I_{n+p N+1}\left(k r_{c}\right)(-1)^{n} P_{n}\left[K_{p N+1}(k r)-\frac{I_{p N+1}(k r)}{h_{p N+1}\left(k r_{t}\right)}\right] e^{i p N \theta} \cos \theta e^{i k z} .
\end{aligned}
$$

Adding the results in Eqs. (A35) and (A56), we have the following solution of Eq. (27) in the region of $r_{c} \leq r \leq r_{i}$ :

$$
\begin{aligned}
\Phi \approx & 2 b_{\perp}\left[K_{1}(k r)-\frac{I_{1}(k r)}{h_{1}\left(k r_{t}\right)}\right] \cos \theta e^{i k z} \\
& +2 N \sum_{p=-\infty}^{\infty} \sum_{n=-\infty}^{\infty} I_{n+p N+1}\left(k r_{c}\right)(-1)^{n} P_{n}\left[K_{p N+1}(k r)-\frac{I_{p N+1}(k r)}{h_{p N+1}\left(k r_{t}\right)}\right] e^{i p N \theta} \cos \theta e^{i k z},
\end{aligned}
$$

where $P_{n}$ is given in Eq. (A54).

We next consider the solution in the $r \leq r_{c}$ region. Here, we have

$$
\phi_{w, m}=\sum_{n=-\infty}^{\infty} \sum_{l=-\infty}^{\infty}\left[P_{n} K_{n+l}\left(k r_{c}\right)+Q_{n} I_{n+l}\left(k r_{c}\right)\right] I_{l}(k r) e^{i l \theta} e^{i l m \Delta} e^{i k z}
$$

and

$$
\phi_{w, m}+\Psi_{w, m}^{\prime}=2 N \sum_{n=-\infty}^{\infty} \sum_{p=-\infty}^{\infty} I_{p N+1}(k r) e^{i p N \theta+i k z} \cos \theta\left[K_{n+p N+1}\left(k r_{c}\right)-(-1)^{n} \frac{I_{n+p N+1}\left(k r_{c}\right)}{h_{p N+1}\left(k r_{t}\right)}\right] P_{n} .
$$

Using these results and Eqs. (A35) and (A36), we obtain the following solution of Eq. (27):

$$
\begin{aligned}
\Phi \approx 2 & b_{\perp}\left[K_{1}(k r)-\frac{I_{1}(k r)}{h_{1}\left(k r_{t}\right)}\right] \cos \theta e^{i k z} \\
& +2 N \sum_{n=-\infty}^{\infty} \sum_{p=-\infty}^{\infty} I_{p N+1}(k r) P_{n}\left[K_{n+p N+1}\left(k r_{c}\right)-(-1)^{n} \frac{I_{n+p N+1}\left(k r_{c}\right)}{h_{p N+1}\left(k r_{t}\right)}\right] e^{i p N \theta} \cos \theta e^{i k z},
\end{aligned}
$$

for $r_{b} \leq r \leq r_{c}$, and

$$
\begin{aligned}
\Phi \approx & 2 b_{\perp}\left[\frac{1}{h_{1}\left(k r_{b}\right)}-\frac{1}{h_{1}\left(k r_{t}\right)}\right] I_{1}(k r) \cos \theta e^{i k z} \\
& +2 N \sum_{n=-\infty}^{\infty} \sum_{p=-\infty}^{\infty} I_{p N+1}(k r) P_{n}\left[K_{n+p N+1}\left(k r_{c}\right)-(-1)^{n} \frac{I_{n+p N+1}\left(k r_{c}\right)}{h_{p N+1}\left(k r_{t}\right)}\right] e^{i p N \theta} \cos \theta e^{i k z},
\end{aligned}
$$

for $r \leq r_{b}$.

The electric field at the beam center is given by

$$
\left.E_{y}\right|_{r=0}=-\left.\frac{\partial \Phi}{\partial r}\right|_{\theta=0} \approx-b_{\perp} k\left[\frac{1}{h_{1}\left(k r_{b}\right)}-\frac{1}{h_{1}\left(k r_{t}\right)}\right] e^{i k z}-N k \sum_{n=-\infty}^{\infty} P_{n}\left[K_{n+1}\left(k r_{c}\right)-(-1)^{n} \frac{I_{n+1}\left(k r_{c}\right)}{h_{1}\left(k r_{t}\right)}\right] e^{i k z} .
$$

If only the monopole solution is considered, i.e., $n=0$,

$$
\left.E_{y}\right|_{r=0} \approx-b_{\perp} k\left[\frac{1}{h_{1}\left(k r_{b}\right)}-\frac{1}{h_{1}\left(k r_{t}\right)}\right] e^{i k z}-N k P_{0}\left[K_{1}\left(k r_{c}\right)-\frac{I_{1}\left(k r_{c}\right)}{h_{1}\left(k r_{t}\right)}\right] e^{i k z},
$$

where

$$
P_{0} \approx \frac{-b_{\perp} h_{0}\left(k \rho_{w}\right)\left\{K_{1}\left(k r_{c}\right)-\left[I_{1}\left(k r_{c}\right) / h_{1}\left(k r_{t}\right)\right]\right\}}{1+h_{0}\left(k \rho_{w}\right)\left\{\sum_{l=-\infty}^{\infty}\left[N \delta_{l,(p N-1)}-1\right] K_{l}\left(k r_{c}\right) I_{l}\left(k r_{c}\right)-N \sum_{p=-\infty}^{\infty} \frac{\left[I_{p N+1}\left(k r_{c}\right)\right]^{2}}{h_{p N+1}\left(k r_{t}\right)}\right\}}
$$


Using the approximations

$$
\sum_{l=-\infty}^{\infty}\left[N \delta_{l,(p N-1)}-1\right] K_{l}\left(k r_{c}\right) I_{l}\left(k r_{c}\right) \approx \frac{N}{2}+\ln \left(k r_{c}\right)-\ln N
$$

and

$$
\sum_{p=-\infty}^{\infty} \frac{\left[I_{p N+1}\left(k r_{c}\right)\right]^{2}}{h_{p N+1}\left(k r_{t}\right)} \approx \frac{1}{2}\left(\frac{r_{c}}{r_{t}}\right)^{2}\left\{1-\frac{1}{N}\left[1+\left(\frac{r_{t}}{r_{c}}\right)^{4}\right] \ln \left[1-\left(\frac{r_{c}}{r_{t}}\right)^{2 N}\right]\right\},
$$

one can show that

$$
P_{0} \approx-\frac{\sigma \bar{d} r_{b}^{2}}{2 \epsilon_{o} r_{c}}\left(1-\frac{r_{c}^{2}}{r_{t}^{2}}\right)\left\{N\left[1-\left(r_{c} / r_{t}\right)^{2}\right]-2 \ln \left(\pi f_{w}\right)+\left[\left(r_{c} / r_{t}\right)^{2}+\left(r_{t} / r_{c}\right)^{2}\right] \ln \left[1-\left(r_{c} / r_{t}\right)^{2 N}\right]\right\}^{-1} .
$$

Therefore,

$$
\left.E_{y}\right|_{r=0} \approx-\frac{\sigma \bar{d} r_{b}^{2}}{2 \epsilon_{o}}\left[\left(\frac{1}{r_{b}^{2}}-\frac{1}{r_{c}^{2}}\right)\left(1-M_{\perp}\right)+\left(\frac{1}{r_{b}^{2}}-\frac{1}{r_{t}^{2}}\right) M_{\perp}\right] e^{i k z},
$$

where $M_{\perp}$ is defined in Eq. (48) in the main text.

\section{APPENDIX B: THE DERIVATION OF PERTURBED FIELD (WITH CERAMIC PIPE)}

\section{The longitudinal perturbed field}

We first consider the solution of Eq. (1) in the absence of wires. In zone I, Eq. (1) has a solution $\left[\left(\sigma / \epsilon_{o} k^{2}\right)+\right.$ $\left.A_{\mathrm{I}} I_{0}(k r)\right] e^{i k z}$, where $A_{\mathrm{I}}$ is a constant to be determined. In zones II, III, and IV, Eq. (1) has the solution in the form of $\left[A_{\nu} I_{0}(k r)+B_{\nu} K_{0}(k r)\right] e^{i k z}$, where $A_{\nu}$ and $B_{\nu}$ are constants, and the subscript $\nu$ represent II, III, or IV. Applying the boundary conditions discussed in the main text, we find

$$
\begin{gathered}
\frac{\sigma}{\epsilon_{o} k^{2}}+A_{\mathrm{I}} I_{0}\left(k r_{b}\right)=A_{\mathrm{II}} I_{0}\left(k r_{b}\right)+B_{\mathrm{II}} K_{0}\left(k r_{b}\right), \\
A_{\mathrm{II}} I_{0}^{\prime}\left(k r_{b}\right)+B_{\mathrm{II}} K_{0}^{\prime}\left(k r_{b}\right)=A_{\mathrm{I}} I_{0}^{\prime}\left(k r_{b}\right), \\
A_{\mathrm{II}} I_{0}\left(k r_{i}\right)+B_{\mathrm{II}} K_{0}\left(k r_{i}\right)=A_{\mathrm{III}} I_{0}\left(k r_{i}\right)+B_{\mathrm{III}} K_{0}\left(k r_{i}\right), \\
A_{\mathrm{II}} I_{0}^{\prime}\left(k r_{i}\right)+B_{\mathrm{II}} K_{0}^{\prime}\left(k r_{i}\right)=\left(\frac{\epsilon}{\epsilon_{o}}\right)\left[A_{\mathrm{III}} I_{0}^{\prime}\left(k r_{i}\right)+B_{\mathrm{III}} K_{0}^{\prime}\left(k r_{i}\right)\right], \\
A_{\mathrm{III}} I_{0}\left(k r_{o}\right)+B_{\mathrm{III}} K_{0}\left(k r_{o}\right)=A_{\mathrm{IV}} I_{0}\left(k r_{o}\right)+B_{\mathrm{IV}} K_{0}\left(k r_{o}\right), \\
A_{\mathrm{III}} I_{0}^{\prime}\left(k r_{o}\right)+B_{\mathrm{III}} K_{0}^{\prime}\left(k r_{o}\right)=\left(\frac{\epsilon_{o}}{\epsilon}\right)\left[A_{\mathrm{IV}} I_{0}^{\prime}\left(k r_{o}\right)+B_{\mathrm{IV}} K_{0}^{\prime}\left(k r_{o}\right)\right],
\end{gathered}
$$

and

$$
A_{\mathrm{IV}} I_{0}\left(k r_{t}\right)+B_{\mathrm{IV}} K_{0}\left(k r_{t}\right)=0 .
$$

Solving Eqs. (B1)-(B7) leads to the following solution of Eq. (1) in zones I and II:

$$
\phi_{b}=\Phi_{\mathrm{I}}=\left\{\frac{\sigma}{\epsilon_{o} k^{2}}-b_{\|} I_{0}(k r)\left[S_{0}+\frac{1}{h_{1}\left(k r_{b}\right)}\right]\right\} e^{i k z}
$$

and

respectively, where

$$
\phi_{b}=\Phi_{\mathrm{II}}=b_{\|}\left[K_{0}(k r)-S_{0} I_{0}(k r)\right] e^{i k z}
$$

$$
\begin{aligned}
S_{l} & =\frac{\epsilon \lambda_{l} I_{l}^{\prime}\left(k r_{i}\right)+\left[\epsilon-\epsilon_{o}-\epsilon_{o} \lambda_{l} h_{l}\left(k r_{i}\right)\right] K_{l}^{\prime}\left(k r_{i}\right)}{\epsilon h_{l}\left(k r_{i}\right) K_{l}^{\prime}\left(k r_{i}\right)-\left[\epsilon_{o}-\lambda_{l}\left(\epsilon-\epsilon_{o}\right) h_{l}\left(k r_{i}\right)\right] I_{l}^{\prime}\left(k r_{i}\right)}, \\
\lambda_{l} & =\frac{1-\hat{\Upsilon} k r_{o} K_{l}\left(k r_{o}\right)\left[I_{l}^{\prime}\left(k r_{o}\right)-h_{l}\left(k r_{t}\right) K_{l}^{\prime}\left(k r_{o}\right)\right]}{\hat{\Upsilon} k r_{o} I_{l}\left(k r_{o}\right)\left[I_{l}^{\prime}\left(k r_{o}\right)-h_{l}\left(k r_{t}\right) K_{l}^{\prime}\left(k r_{o}\right)\right]-h_{l}\left(k r_{t}\right)},
\end{aligned}
$$




$$
\hat{\Upsilon}=1-\left(\epsilon_{o} / \epsilon\right)
$$

$b_{\|}$and $h_{n}(x)$ were defined in Eqs. (A6) and (A7), respectively, and the prime indicates derivative with respect to the argument. Solutions in zones III and IV are similar to Eq. (B9). Since these solutions will not be used directly in calculating the impedance, we will concentrate on the solutions of $\phi_{b}$ in zones I and II only.

The discussion of solving Eq. (1) in the presence of wires will proceed by first considering the solution in the region of $r_{i} \geq r \geq r_{c}$. We assume that each wire induces a potential which has the following multipole expansion in a local coordinate system:

$$
\phi_{w}=\sum_{n=-\infty}^{\infty}\left[C_{\mathrm{II} n} K_{n}(k \rho)+D_{\mathrm{II} n} I_{n}(k \rho)\right] e^{i n \psi} e^{i k z},
$$

where $C_{\mathrm{II} n}$ and $D_{\mathrm{II} n}$ are the unknown quantities to be solved for. In terms of the global coordinate variables, $\phi_{w}$ can be written as

$$
\phi_{w}=\sum_{l=-\infty}^{\infty} \sum_{n=-\infty}^{\infty} I_{n+l}\left(k r_{c}\right)\left[(-1)^{n} C_{\mathrm{II} n} K_{l}(k r)+D_{\mathrm{II} n} I_{l}(k r)\right] e^{i l \theta} e^{i k z}
$$

In zones III and IV, we assume the field due to a wire can be expressed in the global coordinate variables as

$$
\phi_{w}=\sum_{l=-\infty}^{\infty}\left[C_{\nu l} K_{l}(k r)+D_{\nu l} I_{l}(k r)\right] e^{i l \theta} e^{i k z},
$$

where the subscript $\nu$ stands for III or IV. Applying the boundary conditions at $r=r_{i}$ and $r=r_{o}$ together with the condition $\phi_{w}=0$ at $r=r_{t}$, we obtain five simultaneous equations similar to Eqs. (B3)-(B7). Solving these simultaneous equations we find

$$
D_{\mathrm{II} n}=-\sum_{l=-\infty}^{\infty} \sum_{j=-\infty}^{\infty}(-1)^{l} C_{\mathrm{II} l} I_{n+j}\left(k r_{c}\right) S_{j}\left(k r_{t}\right) I_{j+l}\left(k r_{c}\right) .
$$

We call the wire under consideration the 0th wire and number all others by their relative locations with respect to the 0th wire counterclockwise. If $\Psi_{w}$ is the sum of the induced potentials from all other wires near the 0th one, we have

$$
\Psi_{w}=\sum_{l=-\infty}^{\infty} \sum_{n=-\infty}^{\infty}\left[\sum_{\mu=1}^{N-1} e^{i(n+l)(\pi-\mu \Delta) / 2}\right]\left[C_{\mathrm{II} l} K_{l-n}\left(k d_{\mu}\right)+D_{\mathrm{II} l} I_{l-n}\left(k d_{\mu}\right)\right] I_{n}(k \rho) e^{i n \psi} e^{i k z} .
$$

Imposing the condition that the total electric potential,

$$
\Phi=\phi_{b}+\phi_{w}+\Psi_{w}
$$

be zero on the surface of wires, we derive that, on the surface of the 0th wire,

$$
\begin{gathered}
\sum_{l=-\infty}^{\infty}\left[\sum_{\mu=1}^{N-1} e^{i(n+l)(\pi-\mu \Delta) / 2} K_{l-n}\left(k d_{\mu}\right)\right] C_{\mathrm{II} l}+\frac{C_{\mathrm{II} n}}{h_{n}\left(k \rho_{w}\right)}- \\
\sum_{l=-\infty}^{\infty}\left\{\sum_{j=-\infty}^{\infty} I_{n+j}\left(k r_{c}\right) S_{j} I_{j+l}\left(k r_{c}\right)\left[1+(-1)^{n} \sum_{\mu=1}^{N-1} e^{i j \mu \Delta}\right]\right\}(-1)^{l} C_{\mathrm{II} l}+b_{\|}\left[K_{n}\left(k r_{c}\right)-S_{0} I_{n}\left(k r_{c}\right)\right]=0,
\end{gathered}
$$

where use has been made of Eq. (B16).

In the regime of $k \rho_{w} \ll k r_{c}<k r_{t} \ll 1$, one can expand the solution of Eq. (B19) in powers of $h_{n}\left(k \rho_{w}\right)$. If the multipole coupling is neglected, the lowest order solution for $C_{\mathrm{II} n}$ is

$$
C_{\mathrm{II} n} \approx-\frac{b_{\|} h_{n}\left(k \rho_{w}\right)}{G_{\| n}}\left[K_{n}\left(k r_{c}\right)-S_{0} I_{n}\left(k r_{c}\right)\right]
$$

where

$$
G_{\| n}=1+(-1)^{n} h_{n}\left(k \rho_{w}\right) \sum_{\mu=1}^{N-1} e^{-i n \mu \Delta} K_{0}\left(k d_{\mu}\right)-h_{n}\left(k \rho_{w}\right) \sum_{j=-\infty}^{\infty} S_{j}\left[I_{n+j}\left(k r_{c}\right)\right]^{2}\left[1+(-1)^{n} \sum_{\mu=1}^{N-1} e^{i j \mu \Delta}\right],
$$

and $S_{l}$ was defined in Eq. (B10) as well as in Eq. (6) in the main text. The contribution of the potential from all wires can then be found as 


$$
\phi_{w}+\Psi_{w}=N \sum_{p=-\infty}^{\infty} \sum_{n=-\infty}^{\infty}(-1)^{n} C_{\mathrm{II} n} I_{n+p N}\left(k r_{c}\right)\left[K_{p N}(k r)-S_{p N} I_{p N}(k r)\right] e^{i p N \theta} e^{i k z} .
$$

In deriving Eq. (B22), we used Eq. (A20) and the following relation similar to Eq. (A19):

$$
\sum_{n=-\infty}^{\infty} D_{\mathrm{II} n} I_{n+p N}\left(k r_{c}\right)=-\sum_{l=-\infty}^{\infty}(-1)^{l} C_{\mathrm{II} l} S_{p N} I_{l+p N}\left(k r_{c}\right) .
$$

Substituting the potentials in Eqs. (B9) and (B22) into Eq. (B18) yields the following solution of Eq. (1) in the region of $r_{i} \geq r \geq r_{c}$ :

$$
\Phi \approx b_{\|}\left[K_{0}(k r)-S_{0} I_{0}(k r)\right] e^{i k z}+N \sum_{p=-\infty}^{\infty} \sum_{n=-\infty}^{\infty}(-1)^{n} C_{\mathrm{II} n} I_{n+p N}\left(k r_{c}\right)\left[K_{p N}(k r)-S_{p N} I_{p N}(k r)\right] e^{p N \theta} e^{i k z} .
$$

Next, we consider the solution in the region of $r \leq r_{c}$. In this region,

$$
\phi_{w}=\sum_{l=-\infty}^{\infty} \sum_{n=-\infty}^{\infty}\left[C_{\mathrm{II} n} K_{n+l}\left(k r_{c}\right)+D_{\mathrm{II} n} I_{n+l}\left(k r_{c}\right)\right] I_{l}(k r) e^{i l \theta} e^{i k z},
$$

hence the total potential from wires should be

$$
\phi_{w}+\Psi_{w}=N \sum_{p=-\infty}^{\infty} \sum_{n=-\infty}^{\infty} C_{\mathrm{II} n}\left[K_{n+p N}\left(k r_{c}\right)-(-1)^{n} S_{p N} I_{n+p N}\left(k r_{c}\right)\right] I_{p N}(k r) e^{i p N \theta} e^{i k z},
$$

where use was made of Eqs. (A20) and (B23). Note that since the results in Eqs. (B25) and (B26) are derived for the region of $r \leq r_{c}$ they are valid in both zone I and zone II. Substituting Eqs. (B26), (B8), and (B9) into Eq. (B18), the solution of Eq. (1) can be readily found as

$$
\Phi \approx b_{\|}\left[K_{0}(k r)-S_{0} I_{0}(k r)\right] e^{i k z}+N \sum_{p=-\infty}^{\infty} \sum_{n=-\infty}^{\infty} C_{\mathrm{II} n}\left[K_{n+p N}\left(k r_{c}\right)-(-1)^{n} S_{p N} I_{n+p N}\left(k r_{c}\right)\right] I_{p N}(k r) e^{i p N \theta} e^{i k z},
$$

for $r_{c} \geq r \geq r_{b}$, and

$$
\begin{aligned}
\Phi \approx & \left\{\frac{\sigma}{\epsilon_{o} k^{2}}-I_{0}(k r) b_{\|}\left[S_{0}+\frac{1}{h_{1}\left(k r_{b}\right)}\right]\right\} e^{i k z} \\
& +N \sum_{p=-\infty}^{\infty} \sum_{n=-\infty}^{\infty} C_{\mathrm{II} n}\left[K_{n+p N}\left(k r_{c}\right)-(-1)^{n} S_{p N} I_{n+p N}\left(k r_{c}\right)\right] I_{p N}(k r) e^{i p N \theta} e^{i k z},
\end{aligned}
$$

for $r \leq r_{b}$.

\section{The transverse perturbed field}

Following the same procedures in deriving Eqs. (B8) and (B9), we find that the solution of Eq. (A34) in the absence of wires is

$$
\phi_{b}=\Phi_{\mathrm{I}}=b_{\perp}\left[\frac{1}{h_{1}\left(k r_{b}\right)}-S_{1}\right] I_{1}(k r) e^{i \theta} e^{i k z}
$$

in zone I $\left(r \leq r_{b}\right)$, and

$$
\phi_{b}=\Phi_{\mathrm{II}}=b_{\perp}\left[K_{1}(k r)-S_{1} I_{1}(k r)\right] e^{i \theta} e^{i k z},
$$

in zone II $\left(r_{b} \leq r \leq r_{i}\right)$, where $b_{\perp}$ was defined in Eq. (30) as well as in Eq. (A37). Solutions in zones III and IV are similar to Eq. (B30). The following discussions will focus on the solutions of $\phi_{b}$ in zones I and II only.

For the solution including the rf-shielding wires, we first consider the region of $r \geq r_{c}$. As was discussed in Appendix A 2, the system considered here is not axisymmetric so the angular positions of wires need to be taken into account when describing the potential around each wire.

The analysis procedure here will be the same as that in Appendix A 2. Thus, we assume the $m$ th wire induces a potential which can be described in terms of the wire coordinate system variables as

$$
\phi_{w, m}=\sum_{n=-\infty}^{\infty}\left[P_{\mathrm{II} m, n} K_{n}(k \rho)+Q_{\mathrm{II} m, n} I_{n}(k \rho)\right] e^{i n \psi} e^{i k z},
$$

where $P_{\mathrm{II} m, n}$ and $Q_{\mathrm{II} m, n}$ are the unknowns to be solved. Using the addition theorem of Bessel functions, $\phi_{w, m}$ can be expressed in terms of the global coordinate variables as 


$$
\phi_{w, m}=\sum_{n=-\infty}^{\infty} \sum_{l=-\infty}^{\infty} I_{l+n}\left(k r_{c}\right)\left[(-1)^{n} P_{\mathrm{II} m, n} K_{l}(k r)+Q_{\mathrm{II} m, n} I_{l}(k r)\right] e^{i l \theta} e^{i(l-1) m \Delta} e^{i k z}
$$

The contribution from all other $N-1$ wires, $\Psi_{w, m}^{\prime}$, is

$$
\Psi_{w, m}^{\prime}=\sum_{l=-\infty}^{\infty} \sum_{n=-\infty}^{\infty} \sum_{j=0}^{N-1}\left(1-\delta_{j, m}\right) e^{i(n+l)[\pi-(j-m) \Delta] / 2}\left[P_{\mathrm{II} j, l} K_{l-n}\left(k d_{j, m}\right)+Q_{\mathrm{II} j, l} I_{l-n}\left(k d_{j, m}\right)\right] I_{n}(k \rho) e^{i n \psi} e^{i k z},
$$

where $d_{j, m}$ is the distance between the centers of the $m$ th and the $j$ th wires.

Since the total potential on the surface of the $m$ th wire should be zero, one has

$$
\begin{gathered}
\frac{P_{\mathrm{II} m, n}}{h_{n}\left(k \rho_{w}\right)}+Q_{\mathrm{II} m, n}+b_{\perp}\left[K_{n+1}\left(k r_{c}\right)-S_{1} I_{1}\left(k r_{c}\right)\right] e^{i m \Delta}+ \\
\sum_{l=-\infty}^{\infty} \sum_{j=0}^{N-1}\left(1-\delta_{j, m}\right) e^{i(n+l)[\pi-(j-m) \Delta] / 2}\left[P_{\mathrm{II} j, l} K_{l-n}\left(k d_{j, m}\right)+Q_{\mathrm{II} j, l} I_{l-n}\left(k d_{j, m}\right)\right]=0 .
\end{gathered}
$$

For $m=0$, Eq. (B34) becomes

$$
\begin{gathered}
\frac{P_{\mathrm{II} n}}{h_{n}\left(k \rho_{w}\right)}+Q_{\mathrm{II} n}+b_{\perp}\left[K_{n+1}\left(k r_{c}\right)-S_{1} I_{1}\left(k r_{c}\right)\right]+ \\
\sum_{l=-\infty}^{\infty} \sum_{j=0}^{N-1}\left(1-\delta_{j, 0}\right) e^{i(n+l)(\pi-j \Delta) / 2}\left[P_{\mathrm{II} j, l} K_{l-n}\left(k d_{j}\right)+Q_{\mathrm{II} j, l} I_{l-n}\left(k d_{j}\right)\right]=0,
\end{gathered}
$$

where $P_{\mathrm{II} n}=P_{\mathrm{II} 0, n}, Q_{\mathrm{II} n}=Q_{\mathrm{II} 0, n}$, and $d_{j}=d_{j, 0}$. Changing index in Eq. (B34) by letting $j=m+q$ yields

$$
\begin{gathered}
\frac{P_{\mathrm{II} m, n} e^{-i m \Delta}}{h_{n}\left(k \rho_{w}\right)}+Q_{\mathrm{II} m, n} e^{-i m \Delta}+b_{\perp}\left[K_{n+1}\left(k r_{c}\right)-S_{1} I_{n+1}\left(k r_{c}\right)\right]+ \\
\sum_{l=-\infty}^{\infty} \sum_{q=0}^{N-1}\left(1-\delta_{q, 0}\right) e^{i(n+l)(\pi-q \Delta) / 2} e^{-i m \Delta}\left[P_{\mathrm{II} m+q, l} K_{l-n}\left(k d_{q, 0}\right)+Q_{\mathrm{II} m+q, l} I_{l-n}\left(k d_{q, 0}\right)\right]=0 .
\end{gathered}
$$

Equation (B36) has the same form as Eq. (B35). We can infer that $P_{\mathrm{II} n}$ and $P_{\mathrm{II} m, n} e^{-i m \Delta}$ have the same solution, and relations similar to Eqs. (A44)-(A47) also exist for $P_{\mathrm{II} j, l}$ and $P_{\mathrm{II} k}$. Therefore, the requirement of zero total potential on the surfaces of all wires can be reduced to a single equation similar to Eq. (A48):

$$
\begin{aligned}
\sum_{l=-\infty}^{\infty} \sum_{\mu=1}^{N-1} e^{i(n+l)(\pi-\mu \Delta) / 2} e^{i \mu \Delta}\left[P_{\mathrm{II} l} K_{\mathrm{II} l-n}\left(k d_{\mu}\right)+Q_{\mathrm{II} l} I_{l-n}\left(k d_{\mu}\right)\right]+ & \\
\frac{P_{\mathrm{II} n}}{h_{n}\left(k \rho_{w}\right)}+Q_{\mathrm{II} n}+b_{\perp}\left[K_{n+1}\left(k r_{c}\right)-S_{1} I_{n+1}\left(k r_{c}\right)\right] & =0 .
\end{aligned}
$$

We assume that in regions III and IV the field due to the wires has the form of

$$
\phi_{w}=\Phi_{\nu}=2 N \sum_{n=-\infty}^{\infty} \sum_{l=-\infty}^{\infty} I_{n+l}\left(k r_{c}\right)\left[(-1)^{n} P_{\nu n} K_{l}(k r)+Q_{\nu n} I_{l}(k r)\right] e^{i l \theta}\left[\sum_{m=0}^{N-1} e^{i(l-1) m \Delta}\right] \cos \theta e^{i k z},
$$

where $\nu$ stands for III or IV. Applying the boundary conditions at $r=r_{i}$ and $r=r_{o}$ together with the condition $\phi_{w}=0$ at $r=r_{t}$, we obtain five simultaneous equations similar to Eqs. (B3)-(B7). Solving these simultaneous equations determines the constants $P_{\nu n}$ and $Q_{\nu n}$. As a result, we find

$$
Q_{\mathrm{II} m}=-\sum_{n=-\infty}^{\infty}(-1)^{n} P_{\mathrm{II} n} \sum_{l=-\infty}^{\infty} I_{l+n}\left(k r_{c}\right) S_{l} I_{l+j}\left(k r_{c}\right) .
$$

Since the explicit solutions of $\Phi_{I I I}$ and $\Phi_{I V}$ are not involved in deriving the impedance, we concentrate on the explicit solutions in zones I and II in the following. From Eq. (B39), one can show that 


$$
\sum_{l=-\infty}^{\infty} \sum_{\mu=1}^{N-1} e^{i(n+l)(\pi-\mu \Delta) / 2} e^{i \mu \Delta} I_{l-n}\left(k d_{\mu}\right) Q_{\mathrm{II} l}=(-1)^{n+1} \sum_{m=-\infty}^{\infty}(-1)^{m} P_{\mathrm{I} I m} \sum_{j=-\infty}^{\infty} I_{j+m}\left(k r_{c}\right) S_{j} I_{j+n}\left(k r_{c}\right) \sum_{\mu=1}^{N-1} e^{i(j+1) \mu \Delta} .
$$

Using Eq. (B40) and neglecting the multipole coupling, Eq. (B37) can be rewritten as

$$
\begin{aligned}
P_{\mathrm{II} n}\left[1+(-1)^{n} h_{n}\left(k \rho_{w}\right)\right. & \left.\sum_{\mu=1}^{N-1} e^{-i(n-1) \mu \Delta} K_{0}\left(k d_{\mu}\right)\right] \\
-(-1)^{n} P_{\mathrm{II} n} h_{n}\left(k \rho_{w}\right) & \sum_{j=-\infty}^{\infty} S_{j}\left[I_{j+n}\left(k r_{c}\right)\right]^{2}\left[1+(-1)^{n} \sum_{\mu=1}^{N-1} e^{i(j+1) \mu \Delta}\right] \\
& =-b_{\perp} h_{n}\left(k \rho_{w}\right)\left[K_{n+1}\left(k r_{c}\right)-S_{1} I_{n+1}\left(k r_{c}\right)\right],
\end{aligned}
$$

which has the approximate solution

$$
P_{\mathrm{II} n} \approx-b_{\perp} h_{n}\left(k \rho_{w}\right)\left[K_{n+1}\left(k r_{c}\right)-S_{1} I_{n+1}\left(k r_{c}\right)\right] G_{\perp n}^{-1},
$$

where

$$
G_{\perp n}=1+(-1)^{n} h_{n}\left(k \rho_{w}\right)\left\{\sum_{\mu=1}^{N-1} e^{-i(n-1) \mu \Delta} K_{0}\left(k d_{\mu}\right)-\sum_{j=-\infty}^{\infty} S_{j}\left[I_{j+n}\left(k r_{c}\right)\right]^{2}\left[1+(-1)^{n} \sum_{\mu=1}^{N-1} e^{i(j+1) \mu \Delta}\right]\right\} .
$$

Using Eqs. (B32), (A40), and (B39), we can evaluate the potential due to all wires as

$$
\begin{aligned}
\phi_{w, m}+\Psi_{w, m}^{\prime} & =N \sum_{n=-\infty}^{\infty} \sum_{p=-\infty}^{\infty} I_{n+p N+1}\left(k r_{c}\right)\left[(-1)^{n} P_{\mathrm{II} n} K_{p N+1}(k r)+Q_{\mathrm{II} n} I_{p N+1}(k r)\right] e^{i(p N+1) \theta} e^{i k z} \\
& \sim 2 N \sum_{n=-\infty}^{\infty} \sum_{l=-\infty}^{\infty} I_{n+l}\left(k r_{c}\right)\left[(-1)^{n} P_{\mathrm{II} n} K_{l}(k r)+Q_{\mathrm{II} n} I_{l}(k r)\right] e^{i l \theta}\left[\sum_{m=0}^{N-1} e^{i(l-1) m \Delta}\right] \cos \theta e^{i k z} \\
& =2 N \sum_{p=-\infty}^{\infty} \sum_{n=-\infty}^{\infty} I_{n+p N+1}\left(k r_{c}\right)(-1)^{n} P_{\mathrm{II} n}\left[K_{p N+1}(k r)-S_{p N+1} I_{p N+1}(k r)\right] e^{i p N \theta} \cos \theta e^{i k z} .
\end{aligned}
$$

Putting the potentials in Eqs. (B30) and (B44) together gives the following solution of Eq. (27) in the $r_{c} \leq r \leq r_{i}$ region:

$$
\begin{aligned}
\Phi \approx & 2 b_{\perp}\left[K_{1}(k r)-S_{1} I_{1}(k r)\right] \cos \theta e^{i k z} \\
& +2 N \sum_{p=-\infty}^{\infty} \sum_{n=-\infty}^{\infty} I_{n+p N+1}\left(k r_{c}\right)(-1)^{n} P_{\mathrm{IIn}}\left[K_{p N+1}(k r)-S_{p N+1} I_{p N+1}(k r)\right] e^{i p N \theta} \cos \theta e^{i k z},
\end{aligned}
$$

where $P_{\mathrm{II} n}$ is given in Eq. (B42).

Next, we consider the solution in the $r \leq r_{c}$ region. Here we have

$$
\phi_{w, m}=\sum_{n=-\infty}^{\infty} \sum_{l=-\infty}^{\infty}\left[P_{\mathrm{II} n} K_{n+l}\left(k r_{c}\right)+Q_{\mathrm{II} n} I_{n+l}\left(k r_{c}\right)\right] I_{l}(k r) e^{i l \theta} e^{i l m \Delta} e^{i k z},
$$

and

$$
\phi_{w, m}+\Psi_{w, m}^{\prime}=2 N \sum_{n=-\infty}^{\infty} \sum_{p=-\infty}^{\infty} I_{p N+1}(k r) P_{\mathrm{II} n}\left[K_{n+p N+1}\left(k r_{c}\right)-(-1)^{n} S_{p N+1} I_{n+p N+1}\left(k r_{c}\right)\right] e^{i p N \theta} \cos \theta e^{i k z} .
$$

Using these results, we can obtain

$$
\begin{array}{rl}
\Phi \approx 2 & 2 b_{\perp}\left[K_{1}(k r)-S_{1} I_{1}(k r)\right] \cos \theta e^{i k z} \\
& +2 N \sum_{n=-\infty}^{\infty} \sum_{p=-\infty}^{\infty} I_{p N+1}(k r) P_{\mathrm{II} n}\left[K_{n+p N+1}\left(k r_{c}\right)-(-1)^{n} S_{p N+1} I_{n+p N+1}\left(k r_{c}\right)\right] e^{i p N \theta} \cos \theta e^{i k z}
\end{array}
$$

for $r_{b} \leq r \leq r_{c}$, and 


$$
\begin{aligned}
\Phi \approx & 2 b_{\perp}\left[\frac{1}{h_{1}\left(k r_{b}\right)}-S_{1}\right] I_{1}(k r) \cos \theta e^{i k z} \\
& +2 N \sum_{n=-\infty}^{\infty} \sum_{p=-\infty}^{\infty} I_{p N+1}(k r) P_{\mathrm{II} n}\left[K_{n+p N+1}\left(k r_{c}\right)-(-1)^{n} S_{p N+1} I_{n+p N+1}\left(k r_{c}\right)\right] e^{i p N \theta} \cos \theta e^{i k z},
\end{aligned}
$$

for $r \leq r_{b}$.

[1] G. H. Rees, in Proceedings of the 1983 Particle Accelerator Conference, Santa Fe, NM [IEEE Trans. Nucl. Sci. 30, 3044 (1983)].

[2] Los Alamos National Laboratory Report No. LA-10720MS, 1986.

[3] "Kaon Factory Study: Accelerator Design Report," TRIUMF-Kaon Project, 1990.

[4] "AUSTRON Feasibility Study," Im Auftrage des Bundesministeriums für Wissenschaft und Forschung Report, edited by P. Bryant, M. Regler, and M. Schuster, 1994.

[5] KEK Report No. 97-3, JHF 97-1, 1997.

[6] B. W. Zotter and S. A. Kheifets, Impedances and Wakes in High-Energy Particle Accelerators (World Scientific, River Edge, New Jersey, 1998).

[7] R.L. Gluckstern, lecture note in U.S. Particle Accelerator School, Phoenix, AZ, 2000 (unpublished).

[8] T.F. Wang, CERN Internal Report No. CERN/PS-94-08, 1994.
[9] T. F. Wang, in Workshop on Space Charge Physics in High Intensity Hadron Rings, edited by A. U. Luccio and W. T. Weng, AIP Conf. Proc. No. 448 (AIP, New York, 1998), p. 286.

[10] T.F. Wang and R.L. Gluckstern, in Proceedings of the 1999 Particle Accelerator Conference, New York (IEEE, Piscataway, NJ, 1999), Vol. 4, p. 2876.

[11] S. S. Kurennoy, in Workshop on Instabilities of High Intensity Hadron Beams in Rings, edited by T. Roser and S. Y. Zhang, AIP Conf. Proc. No. 496 (AIP, New York, 1999), p. 361 .

[12] See Chaps. 16 and 17 of Ref. [7].

[13] R. Gluckstern and B. Zotter, Phys. Rev. ST Accel. Beams 4, 024402 (2001).

[14] MAFIA, release 4.025 (CST, Darmstadt, Germany 2000).

[15] The addition theorem of Bessel functions can be found in almost any textbook or mathematical table discussion about Bessel functions. See, for example, M. Abramowitz and I. A. Stegun, Handbook of Mathematical Functions (U.S. National Bureau of Standards, Department of Commerce, Washington, DC, 1964). 